This document is confidential and is proprietary to the American Chemical Society and its authors. Do not copy or disclose without written permission. If you have received this item in error, notify the sender and delete all copies.

\title{
Proton transfer and structure-specific fluorescence in hydrogen bond-rich protein structures
}

\begin{tabular}{|r|l|}
\hline Journal: & Journal of the American Chemical Society \\
\hline Manuscript ID & ja-2015-110125.R3 \\
\hline Manuscript Type: & Article \\
\hline Date Submitted by the Author: & $29-J a n-2016$ \\
\hline Complete List of Authors: & $\begin{array}{l}\text { Pinotsi, Dorothea; Cambridge University, Chemical Engineering } \\
\text { Grisanti, Luca; the "Abdus Salam" ICTP, } \\
\text { Mahou, Pierre; Cambridge University, Chemical Engineering } \\
\text { Gebauer, Ralph; International Center for Theoretical Physics } \\
\text { Kaminski, Clemens; University of Cambridge, Dept. of Chemical } \\
\text { Engineering } \\
\text { Hassanali, Ali; International Center for Theoretical Physics, Condensed } \\
\text { Matter and Statistical Physics Section } \\
\text { Kaminski Schierle, Gabriele; University of Cambridge, }\end{array}$ \\
\hline
\end{tabular}

SCHOLARONE

Manuscripts 


\title{
Proton transfer and structure-specific fluorescence in hydrogen bond-
} rich protein structures

\author{
Dorothea Pinotsi ${ }^{1}$, Luca Grisanti ${ }^{2}$, Pierre Mahou ${ }^{1}$, Ralph Gebauer ${ }^{2}$, Clemens F. Kaminski ${ }^{1}$, Ali \\ Hassanali $^{2^{*}}$, Gabriele S. Kaminski Schierle ${ }^{1^{*}}$ \\ ${ }^{1}$ Department of Chemical Engineering and Biotechnology, University of Cambridge, Pembroke \\ Street, Cambridge CB2 3RA, UK; ${ }^{2}$ International Centre for Theoretical Physics, Strada Costiera 11, \\ 34151 Trieste, Italy. \\ *To whom the correspondence should be addressed: ahassana@ictp.it and gsk20@cam.ac.uk
}

\begin{abstract}
:
Protein structures which form fibrils have recently been shown to absorb light at energies in the near UV range and to exhibit a structure-specific fluorescence in the visible range even in the absence of aromatic amino acids. However, the molecular origin of this phenomenon has so far remained elusive. Here, we combine $a b$ initio molecular dynamics simulations and fluorescence spectroscopy to demonstrate that these intrinsically fluorescent protein fibrils are permissive to proton transfer across hydrogen bonds which can lower electron excitation energies and thereby decrease the likelihood of energy dissipation associated with conventional hydrogen bonds. The importance of proton transfer on the intrinsic fluorescence observed in protein fibrils is signified by large reductions in the fluorescence intensity upon either fully protonating, or deprotonating, the fibrils at $\mathrm{pH}=0$ or $\mathrm{pH}=14$, respectively. Thus, our results point to the existence of a structure-specific fluorophore that does not require the presence of aromatic residues or multiple bond conjugation that characterise conventional fluorescent systems. The phenomenon may have a wide range of implications in biological systems and in the design of self-assembled functional materials.
\end{abstract}

\section{Introduction:}

Understanding the mechanisms associated with the formation of protein fibrils, the kinetic aspects of their aggregation in different environments and, more recently, their optical properties, is a subject of intense investigation $^{1-9}$. The three-dimensional (3D) arrangement of protein fibrils consists to a large extent of $\beta$-sheets ${ }^{10,11}$ which are stabilized by a dense network of hydrogen bonds (H-bonds). Upon fibrillization such structures reach the thermodynamically most stable state and the ensuing H-bonds interconnecting the $\beta$-sheets have been shown to be stronger than those found in any other folded $\operatorname{protein}^{12}$. 
In recent work it was found that protein fibrils absorb light at wavelengths $\lambda>360 \mathrm{~nm}$ with subsequent fluorescence emission that is red shifted to $\lambda>400 \mathrm{~nm}$ leading to fluorescence lifetimes on the order of 1-3 nsec ${ }^{1,2,4,5,7,9,13}$. This fluorescence phenomenon has been attributed to either the dipolar coupling between the excited states of aromatic residues ${ }^{3,4,8}$ or linked to the presence of the dense network of H-bonds interconnecting the $\beta$-sheet strands. Recent evidence points to the latter since fluorescence is also observed from fibrils free of any aromatic residues ${ }^{1,2,5,13}$. This observation is puzzling since absorption of photons and subsequent fluorescence in biological fluorophores is typically associated with the presence of aromatic residues or conjugated bonds that lead to electron delocalization $^{14-16}$.

The role of the dense H-bond network in determining the optical properties of $\beta$-sheet rich structures has been a subject of interest since the 1950's. Schauenstein proposed the existence of a peptenol chromophore in fibrillar silk proteins, which was thought to involve the movement of protons along the peptide backbone and thus to give rise to electron delocalization ${ }^{17}$. Delocalized electronic densities in H-bonds have furthermore been reported to occur in some organic molecules, such as polypeptides and enzymes ${ }^{18,19}$. Several theoretical studies on model organic systems have also shown that the displacement of protons along specific interaction coordinates, such as H-bonds, can be strongly coupled to photo-physical processes ${ }^{20-22}$. In addition to the coupling of proton motion to delocalized electronic densities, there is also a growing body of evidence on the importance of nuclear quantum effects in several systems. In particular, since protons are light particles, the role of zero-point energy has been found to significantly alter structural and electronic properties of hydrogen bonded systems $^{23-25}$. We, and others, have thus hypothesized that charge delocalization in the extensive $\mathrm{H}$ bond network present in protein fibrils may be responsible for the emergence of the fluorescence observed in these structures. However, evidence for this effect has remained elusive until this point.

In this work we combine state-of-the-art large-scale first principles simulations of small protein fibrils, such as formed by amyloid proteins, and quantitative fluorescence spectroscopy experiments to show that there is indeed charge delocalization, and in particular proton transfer, occurring in $\mathrm{H}$ bond-rich structures independent of aromatic residues. This suggests a novel mechanism by which fluorescence may occur in the absence of any classical $\pi$-conjugation system. Our simulations show that protons in the ground state can easily transfer along H-bonds formed between the N- and Ctermini of these protein fibrils (see schematic in Fig. 1). Furthermore, we find that this process can facilitate absorbance of photons at lower excitation energies and perhaps more importantly decrease the likelihood of energy dissipation associated with conventional H-bonds. We further provide experimental proof that proton transfer is directly coupled to the intrinsic fluorescence phenomenon, as protein fibrils in either a fully protonated or deprotonated form undergo significant changes in their optical properties. 
a)

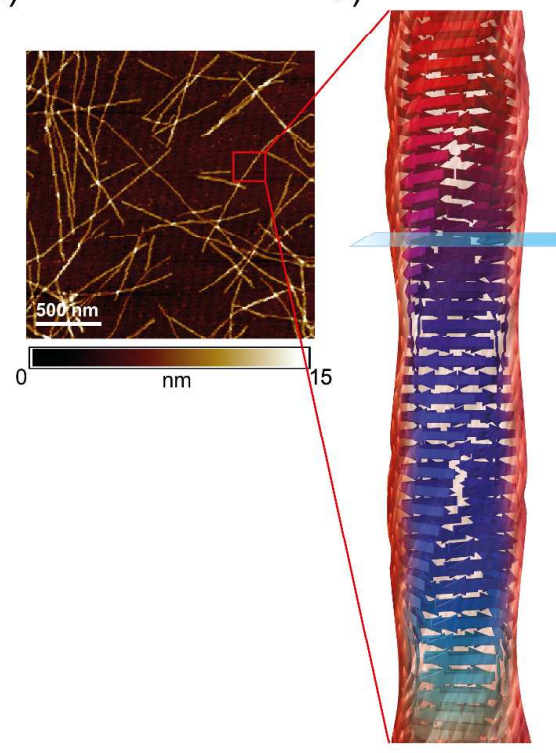

c)

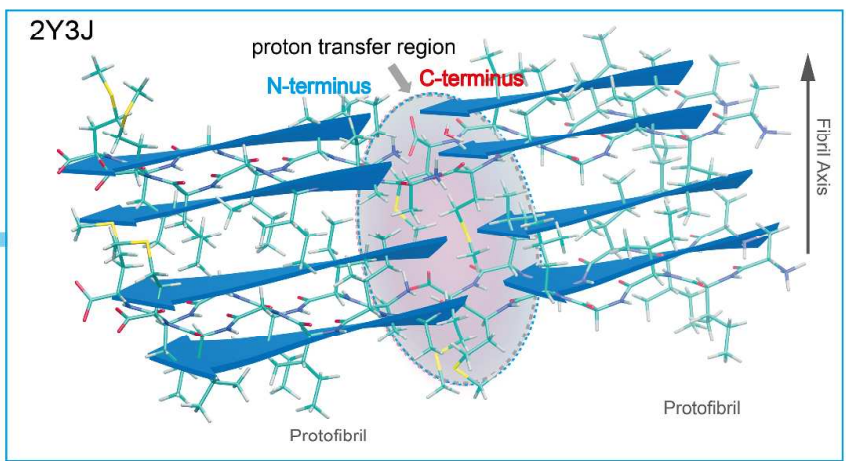

d)

i) $2 \mathrm{Y} 3 \mathrm{~L}$

ii) $2 \mathrm{Y} 2 \mathrm{~A}$
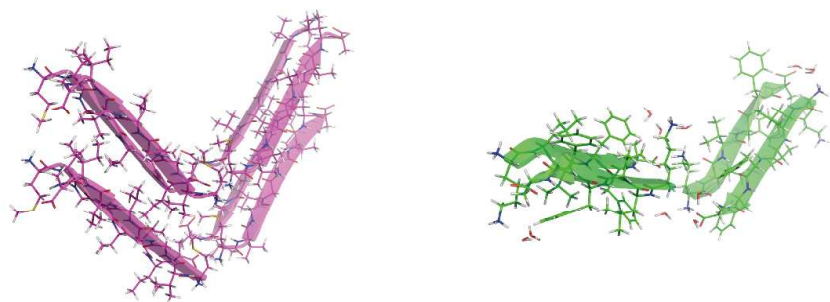

Figure 1: Structural arrangements of protein fibrils studied: a) AFM image of protein fibrils, scale bar: $500 \mathrm{~nm}$. b) Schematic view of the three dimensional peptide arrangement in a protein fibril such as formed by amyloid proteins. The fibril shown consists of four protofilaments twisted around a common axis. The depicted protofilaments are formed by stacks of $B$ strands. A cross-section of the protein fibril is shown. c) Magnified depiction of the cross-section indicated in (b) showing sections from two adjacent protofilaments: the peptide chains (parallel B-strands) correspond to the unit cell of 2Y3J (used as a model system for the simulations). The shaded ellipsoid depicts the region in which our simulations predict proton transfer to occur frequently. d) Peptide arrangement of the unit cells of the two other model protein fibrils studied: 2Y3L (left) and $2 Y 2 A$ (right). Note that in contrast to $2 Y_{3} \mathrm{~J}$ the $B$-strands within the unit cells of $2 \mathrm{Y} 3 \mathrm{~L}$ (i) and $2 \mathrm{Y} 2 \mathrm{~A}$ (ii) are in an antiparallel conformation.

\section{Methods:}

\section{Computational section:}

The computational modelling of the protein fibrils and determination of the optical spectra involved two main stages. We used first principles simulations for which the electronic degrees of freedom were explicitly treated to perform molecular dynamics simulations in the electronic ground state. We then used these simulations to extract configurations from which static absorption spectra could be determined. For certain cases we also performed full quantum simulations of the nuclei for which zero-point energy and tunnelling effects were explicitly accounted for. Since the simulation of the entire protein fibrils would be computationally intractable, we used model systems based on the known crystal structures of representative amyloid forming peptides or fragments of the actual peptides used in the experiments. The model systems correspond to the crystal structures of $2 \mathrm{Y} 3 \mathrm{~J}$, 2Y3L and 2Y2A (AIIGLM, MVGGVVIA and KLVFFA), respectively in the protein database (PDB). More details on these model systems are given in the main text.

\section{Equilibrium Ground State simulations}


The ab initio Molecular Dynamics (AIMD) simulations of the ground-state were performed using the Quickstep algorithm which is part of the $\mathrm{CP} 2 \mathrm{~K}$ package ${ }^{26}$. In these calculations, ab initio BornOppenheimer molecular dynamics is used for the propagation of classical nuclei. A convergence criterion of $5^{*} 10-7$ a.u. was used for the optimization of the wave function. Using the Gaussian and plane wave methods, the wave function was expanded in the Gaussian double zeta valence polarized (DZVP) basis set. An auxiliary basis set of plane waves was used to expand the electron density up to a cut-off of $300 \mathrm{Ry}$. For the simulations we used the gradient correction ${ }^{27}$ to the local density approximation and Goedecker-Teter-Hutter pseudopotentials for treating the core electrons ${ }^{28}$. Unless stated otherwise, all calculations employed the Becke-Lee-Yang-Parr (BLYP) functional with the D3(0) Grimme dispersion corrections for the Van-der-Waals interactions ${ }^{29}$. The structures obtained from the PDB databank were first structurally optimized at $0 \mathrm{~K}$ using the Broyden-FletcherGoldfarb-Shanno (BFGS) minimizer ${ }^{30}$. The finite temperature simulations were conducted within the canonical NVT ensemble using the canonical-sampling velocity-rescaling thermostat method ${ }^{31}$. The quantum simulations were performed using a method that combines path-integrals with Langevin thermostating, permitting the usage of a small number of beads (PIGLET) ${ }^{32}$. We employed 6 beads for these calculations which has been shown to give good convergence for statistical properties in liquid water ${ }^{23}$. The total lengths of the classical simulations for $2 \mathrm{Y} 3 \mathrm{~J}, 2 \mathrm{Y} 3 \mathrm{~L}$ and $2 \mathrm{Y} 2 \mathrm{~A}$ were $23 \mathrm{ps}, 13$ ps and $18 \mathrm{ps}$, respectively. The PIGLET simulations of $2 \mathrm{Y} 2 \mathrm{~A}$ were run for $\sim 6 \mathrm{ps}$. Note that $2 \mathrm{Y} 3 \mathrm{~J}$, 2Y3L and 2Y2A consist of a total of 758, 888, and 476 atoms, respectively, which makes AIMD simulations computationally demanding.

\section{Excited state calculations}

Excited state calculations were performed using Time-Dependent Density Functional Theory (TDDFT) implemented in the freely available Quantum-Espresso software package ${ }^{33}$. The method employs a Liouville-Lanczos formalism to compute optical spectra directly over a wide spectral range, avoiding the need for numerically complex calculation of single excited states. Details of this approach have been presented elsewhere ${ }^{33,34}$. A plane-wave basis set was used and the electron-ion interactions were taken into account via Vanderbilt ultrasoft pseudopotentials. The ground-state wave functions were determined at the $\Gamma$ point of the Brillouin zone. All calculations employed the BeckeLee-Yang-Parr (BLYP) functional with periodic boundary conditions; a kinetic energy cut-off of 25 Ry for the wave functions and of 300 Ry for the charge density were chosen. For the spectra, an intrinsic bandwidth was set to $0.01 \mathrm{Ry}(\sim 0.12 \mathrm{eV})$. A spectral analysis was performed using the second derivative of the spectral line shape in order to estimate the excited state energies: local minima in the 2nd derivative of the spectra correspond to local maxima in the spectra, and hence to an excited state. Room temperature excitation spectra calculations were computed on frames uniformly sampled along the room temperature AIMD simulations. Finally, excited state electronic densities 
were computed according to turbo lanczos module: the 3D responses (one for each dipolar component) were summed to obtain an overall electronic response density.

\section{Experimental section:}

\section{Materials}

All chemicals for the preparation of buffers including heparin were purchased from Sigma Aldrich (Dorset, UK). The 'synthetic' analogs of human $A \beta_{1-42}$ and $A \beta_{33-42}$ peptides (Bachem, Merseyside, UK) were prepared by sequential treatment with trifluoroacetic acid (TFA) and hexafluoroiso-propanol (HFIP) to remove small aggregates and stored as lyophilized film in Eppendorf tubes at $-20{ }^{\circ} \mathrm{C}{ }^{35}$. Samples were freshly thawed just before each set of experiments. The Tau construct K18 (I260C/C291A/C322A) was prepared as described in Ref. 7.

\section{Conversion of soluble polypeptides into fibrils}

$100 \mu \mathrm{M}$ lyophilized human $\mathrm{A} \beta_{1-42}$ peptide was dissolved in either 1x Phosphate-Buffered Saline (PBS) (pH 7.4) or in varying concentrations of $\mathrm{HCl}$ or $\mathrm{NaOH}$ to adjust the $\mathrm{pH}$, and incubated at $37{ }^{\circ} \mathrm{C}$ for 7 days, to ensure that in all solutions there was sufficient time for the consumption of the monomeric protein and the conversion to fibrils. The final fibrillar sample was then diluted to $50 \mu \mathrm{M}$ using one of the following: $1 \mathrm{x}$ PBS, $0.01 \mathrm{M}$ or $1 \mathrm{M} \mathrm{HCl}$, or $1 \mathrm{M} \mathrm{NaOH}$. Lyophilized $\mathrm{A} \beta_{33-42}$ was initially dissolved in $1 \% \mathrm{NH}_{4} \mathrm{OH}$ at $2 \mathrm{mM}$ and diluted to $100 \mu \mathrm{M}$ in either $1 \mathrm{x}$ PBS or in $\mathrm{HCl}$ and $\mathrm{NaOH}$ at the concentrations stated above, depending on which $\mathrm{pH}$ was required. The sample was centrifuged $(13400 \mathrm{~g}, 10 \mathrm{~min})$ to remove any undissolved peptide and the soluble fraction was incubated at $37{ }^{\circ} \mathrm{C}$ for 7 days. The final fibrillar sample was then diluted to $50 \mu \mathrm{M}$ using the corresponding solvent. K18 (I260C/C291A/C322A) Tau (10 $\mu \mathrm{M}$ in $50 \mathrm{mM}$ ammonium acetate buffer, $\mathrm{pH}$ 7.2) was incubated for $24 \mathrm{~h}$ at $37^{\circ} \mathrm{C}$ under quiescent conditions in the presence of $2.5 \mu \mathrm{M}$ heparin (MW3000, Sigma-Aldrich). All buffers were made with either Milli-Q water, or with deuterium oxide $\left(\mathrm{D}_{2} \mathrm{O}\right)$ (Sigma-Aldrich) and were filtered with $0.2 \mu \mathrm{M}$ filters (Millipore Limited, Watford, UK) before use. In experiments requiring resuspensions of fibrils in different solvents, the fibrillar sample was first centrifuged (10000g, $30 \mathrm{~min})$ and the soluble fraction was then removed and replaced with an equal volume of the solvent.

\section{Spectroscopy and Confocal microscopy}

Excitation and emission spectra of bulk solutions containing various amyloid fibrils were taken at $25^{\circ} \mathrm{C}$ using an F-4500 fluorescence spectrophotometer (Hitachi High Tech, Tokyo, Japan). The excitation spectra were measured with the detection wavelength fixed at $450 \mathrm{~nm}$. The emission spectra were measured with the excitation wavelength fixed at $365 \mathrm{~nm}$. For all measurements, excitation and emission slit widths were set to yield bandwidths of 5 and $10 \mathrm{~nm}$, respectively. The 
PMT voltage was set at $700 \mathrm{~V}$; the scan speed was $60 \mathrm{~nm} / \mathrm{min}$. Microscopic images of protein fibrils were obtained by recording their intrinsic fluorescence on a confocal microscope (Leica TCS SP5, Leica Microsystems GmbH, Wetzlar, Germany) using a $405 \mathrm{~nm}$ diode laser as the excitation source and a $63 x, 1.4$ NA oil immersion objective. For spectral measurements of individual fibrils on the confocal microscope the emission bandwidth used was set to $20 \mathrm{~nm}$. It was verified that photobleaching was insignificant during image and spectrum acquisition. At least ten independent spectra were recorded of the intrinsic fluorescence for every experimental condition. Each experiment was furthermore repeated 3 times using 3 different preparations of fibrils which were formed under the same conditions. Spectra were fitted with Lorentzian functions (OriginLab, Northampton, USA), the peak wavelengths were determined and values from all different runs were then averaged. Statistical analyses were performed using Prism (GraphPad Software, Inc., La Jolla, CA, USA).

\section{Fluorescence lifetime imaging microscopy (FLIM)}

All fluorescence lifetime images were recorded on a home built confocal microscopy platform based on a confocal microscope scanning unit (Olympus FluoView 300) coupled with an Olympus IX70 inverted microscope frame (Olympus UK, Essex, UK). Details can be found in Ref. 5. In summary, we used a pulsed laser excitation at $405 \mathrm{~nm}$ (sub 10 ps pulses at $40 \mathrm{MHz}$ repetition rate) stemming from a supercontinuum source (SC 450, Fianium Ltd., Southampton, UK). The following excitation and emission filters were used: a 400/40 nm and a 475/50 nm bandpass filter (FF01-400/40 and FF02475/50, Semrock, USA) respectively.

\section{Two-photon fluorescence measurements}

Two-photon measurements were performed using a titanium sapphire laser $(\lambda=750-950 \mathrm{~nm}$, Mai Tai, Spectra-Physics, USA) coupled to a confocal microscope (Aberrior Instruments, Germany). The laser beam was focused onto the sample by an oil immersion objective (100x 1.4 NA, Olympus, USA) and the excitation power was adjusted using an attenuator consisting of a motorized half-wave plate (AHWP05M-980, Thorlabs) and a polarizer (05FC16PB.5, Newport). The two-photon fluorescence was separated from the excitation beam with a combination of a dichroic mirror (T735spxr, Chroma Technology, USA) and a bandpass filter (FF01-550/88, Semrock, USA) and collected with an avalanche photodiode (SPCM-AQRH, Excelitas Technologies, USA) and an open pinhole. Depending on the amount of collected counts we used integration times ranging from 100 to $500 \mu \mathrm{s}$. Synchronization of the galvanometer mirrors and detector was performed using the Imspector Image Acquisition software (Andreas Schönle, Abberior Instruments, Göttingen, Germany) and all other peripheral devices, including motorized half-wave plate and shutter were controlled using customwritten LabVIEW (National Instruments, Austin, USA) code. 
Two-photon fluorescence signals from $A \beta_{1^{-42}}$ and $A \beta_{33^{-42}}$ fibrils were confirmed each time by the presence of corresponding one-photon fluorescence signals when excited at $405 \mathrm{~nm}$. The intensity dependence was measured through acquisition of a stack of images with increasing excitation intensity. The mean of the collected fluorescence signal $(\langle F\rangle)$ was then plotted as function of the estimated average intensity at the focus of the objective and fitted using Matlab (MathWorks, USA) with a linear regression: $\log (\langle F\rangle)=a \times \log (\langle I\rangle)+b$ in which the coefficient $a$ represents the order of the process. For the measurement of the relative two-photon absorption, a series of images were acquired for excitation wavelengths ranging from 750 to $950 \mathrm{~nm}$ in $20 \mathrm{~nm}$ steps. The average power was kept constant over the whole spectral range and measured precisely for each excitation wavelength. The average fluorescent signal $\langle F\rangle$ was then normalized with the average measured excitation power $\langle P\rangle$ and wavelength $\lambda$ as follows: $\langle F\rangle \lambda /\langle P\rangle^{2}$ and the excitation spectra were plotted. All measurements were repeated on three different samples for each fibril type $\left(A \beta_{1^{-42}}\right.$ and $\left.A \beta_{33^{-42}}\right)$ and found to be consistent with one another.

\section{Results and Discussion:}

\section{Proton transfer occurs in protein fibrils as shown by ab initio molecular dynamics simulations}

Previous reports have shown that proton tunnelling can lead to the formation of delocalized electronic states in systems that feature extended H-bond networks such as $\mathrm{DNA}^{36}$ and certain proteins ${ }^{24}$. Recently, Boxer and co-workers have also found significant nuclear quantum effects in the Green Fluorescent Protein (GFP), which affects the structural fluctuations of protons ${ }^{37}$. To probe deeper into the structural, chemical and electronic properties of the H-bonds formed in $\beta$-sheet rich protein fibrils and their link to fluorescence, we employed ab initio molecular dynamics (AIMD) simulations ${ }^{26,38}$ (see Methods Section) using the small fibril forming peptides AIIGLM, MVGGVVIA and KLVFFA ${ }^{39}$ as model systems. These correspond to the following different segments of the Amyloid $\beta\left(\mathrm{A} \beta_{42}\right)$ peptide, namely $A \beta_{30-35}, A \beta_{35-42}$ (both void of aromatic residues), and $A \beta_{16-21}$ (containing two phenylalanines). Their corresponding protein database (PDB) crystal structures are $2 \mathrm{Y} 3 \mathrm{~J}, 2 \mathrm{Y} 3 \mathrm{~L}$, and $2 \mathrm{Y} 2 \mathrm{~A}$, respectively. All form $\beta$-sheet rich fibrils which are composed of several stacks of $\beta$-strands, the so-called protofibrils ${ }^{11}$ (Fig. 1). Because of its size and associated computational cost it is not possible to conduct AIMD simulations of the entire $A \beta_{42}$ peptide. The current systems were chosen to represent the diversity in structural morphologies found experimentally in full-length $A \beta$ fibrils and, although large with more than 700 atoms in the unit cell, remain computationally tractable for our approach. In 2Y3J the $\beta$-sheets are arranged in a parallel fashion with respect to each other (Fig. 1c), while for $2 \mathrm{Y} 3 \mathrm{~L}$ and $2 \mathrm{Y} 2 \mathrm{~A}$ they are arranged in an anti-parallel fashion (Fig. 1d (i) and (ii) respectively). We note that in each case the $\mathrm{N}$-terminus of one $\beta$-strand resides in close proximity to the $\mathrm{C}$-terminus of the neighbouring $\beta$-strand and the strands are bridged by $\mathrm{H}$-bonds which feature slightly different bonding patterns for the three structures studied. 
a)

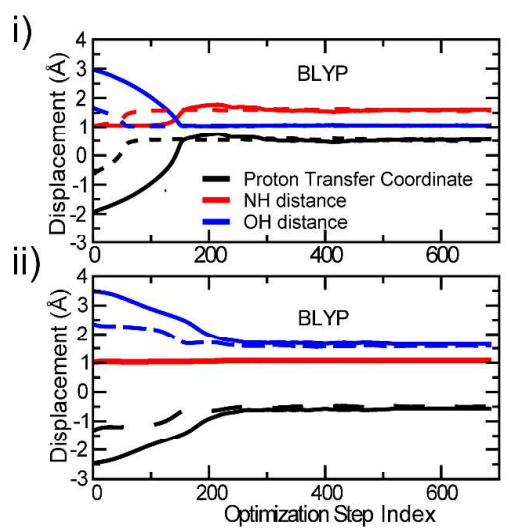

b)

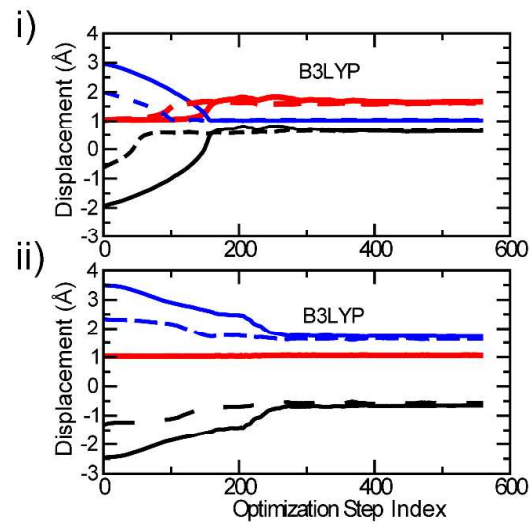

c)

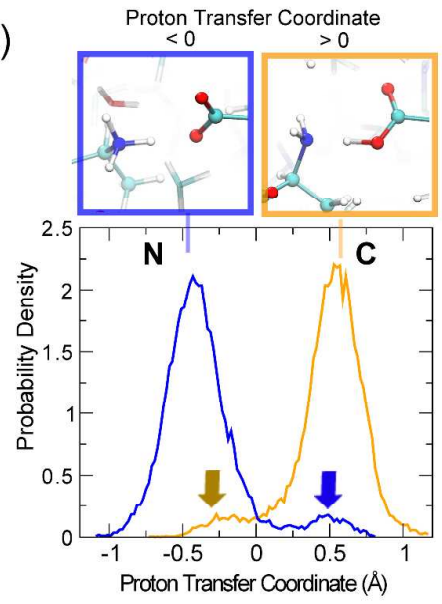

Figure 2: a) i) The evolution of various proton displacements during simulations at $0 \mathrm{~K}$ of $2 \mathrm{Y}_{3} \mathrm{~J}$ (starting from the experimental crystal structure as input) applying the BLYP functional. In particular, we focus on the proton transfer events that occur along two different hydrogen bonds (H-bonds) in the system (solid and dashed lines). The red curves correspond to the $\mathrm{N}-\mathrm{H}$ distance while the blue curves correspond to the $\mathrm{O}-\mathrm{H}$ distance where $\mathrm{H}$ is the transferring proton. The black curves are the proton transfer coordinates. When the $\mathrm{N}-\mathrm{H}$ bond length increases, the $\mathrm{O}-\mathrm{H}$ bond length decreases and the proton is transferred from the $\mathrm{N}$ - to the $\mathrm{C}$-terminus. When the proton transfer coordinate is $\mathrm{O}$, the $\mathrm{N}$-H bond length is equal to that of the $\mathrm{O}-\mathrm{H}$ bond and hence the proton is equally shared. ii) Same plot but here no proton transfer occurs. b) Same results as shown in (a) but derived by using the hybrid functional B3LYP to provide a comparison: the results are the same as in (a) and thus independent of the choice of functional. c) The thermal distribution of 2 selected proton transfer coordinates in $2 Y_{3} \mathrm{~J}$ obtained performing finite temperature (300 K) simulations. These probability density distributions are characterized by a double-well character for which the proton can either be stabilized at the $\mathrm{N}$-terminus or at the Cterminus. The arrows indicate the presence of additional small peaks giving the double-well character to the potential.

Fig. 2a (i) shows the evolution of the lengths of two different $\mathrm{N}-\mathrm{H}$ and $\mathrm{O}-\mathrm{H}$ bonds during the simulation: the N-H bond increases in length from $1 \AA$ to about $1.5 \AA$ (solid and dashed red lines) while the $\mathrm{O}-\mathrm{H}$ bond decreases in length (solid and dashed blue lines) thus enabling a complete proton transfer from the N-terminus to one of the carboxylate oxygen atoms. The solid and dashed lines correspond to two different $\mathrm{H}$-bonds in the fibril for which proton transfer occurs at $0 \mathrm{~K}$. The evolution of the proton transfer coordinate is also shown (black line), defined as the difference in distance between the N-H bond and $\mathrm{O}-\mathrm{H}$ bond for which $\mathrm{H}$ refers to the transferring proton. Thus, when the $\mathrm{N}-\mathrm{H}$ and $\mathrm{O}-\mathrm{H}$ bonds are equal in length, the proton transfer coordinate is zero, meaning that the proton is equally shared between both termini. A complete proton transfer results in the transformation of the $\mathrm{NH}_{3}{ }^{+}---\mathrm{COO}^{-}$ion pair to $\mathrm{NH}_{2}---\mathrm{COOH}$ (see also SI movie 1 and Suppl. Fig. S1 for the $0 \mathrm{~K}$ simulation). 
a)

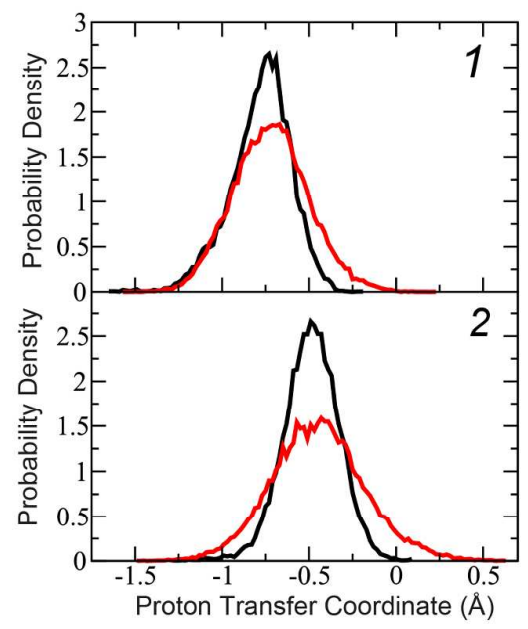

b)

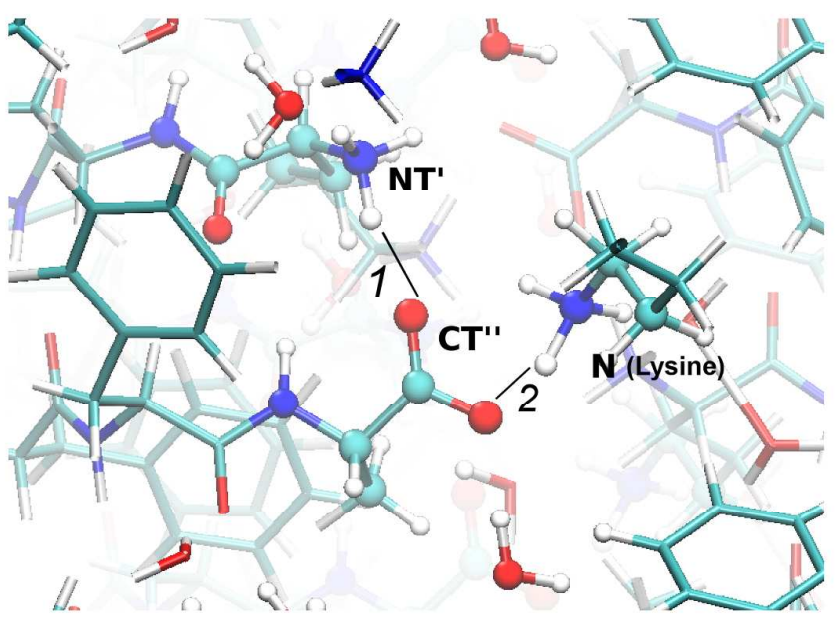

Figure 3: a) Top and bottom panels: probability density distributions of the proton transfer coordinates obtained from simulations of 2 different $\mathrm{H}$-bonds for the 2Y2A model fibrils. The $\mathrm{H}$-bonds correspond to bridges between the $\mathrm{N}$ - and $\mathrm{C}$ termini (1) or between a lysine side-chain and the C-terminus (2), as indicated in panel (b). b) A snapshot illustrating the two hydrogen bonds (1 and 2) in which the C-terminus is involved. The H-bonds between the lysine and the C-terminus tend to be shorter than those between the $\mathrm{N}$ - and $\mathrm{C}$-termini. However, in both situations the proton transfer distributions obtained from the quantum simulations (red) are significantly more delocalized compared to the classical ones (black). Quantum fluctuations of the protons lead to situations where there is a significantly higher probability of finding the proton equally shared, i.e. the proton transfer coordinate is close to 0.

On the other hand, in Fig. 2a (ii) we show the evolution of the same coordinates $(\mathrm{N}-\mathrm{H}, \mathrm{O}-\mathrm{H}$ and the proton transfer coordinate) for two N-C termini sites for which proton transfers do not occur. In this case we observe that the N-H bond length remains at around $1 \AA$ and the O-H bond length converges to $\sim 1.6 \AA$. In this scenario the proton transfer coordinate remains negative throughout the simulation. These optimization simulations therefore show that there are sites in the protein fibril which are protonated either as $\mathrm{NH}_{3}{ }^{+}-\mathrm{COO}^{-}$ion pairs, or as $\mathrm{NH}_{2}-\mathrm{COOH}$. We ascertained that the predicted proton transfer events were not sensitive to the choice of functional applied and thus repeated the $0 \mathrm{~K}$ simulation for $2 \mathrm{Y} 3 \mathrm{~J}$ with a more accurate, but computationally more costly, hybrid functional the B3LYP as implemented in the CP2K code $e^{40,41}$. Using B3LYP we confirmed that the protons are indeed labile and move across the N-C-termini H-bonds, as illustrated in Fig. 2b (i). Similarly, using B3LYP the proton can also be stabilized on the N-terminus as seen in Fig. $2 b$ (ii).

In order to investigate the role of temperature on the fluctuations of protons and to extract information on the underlying free energy profiles, we performed AIMD simulations at $300 \mathrm{~K}$. Fig. 2c shows the probability density distributions of the proton transfer coordinates of two different H-bonds. The peak of the probability density distribution resides at either a negative proton transfer coordinate, implying that the proton is localized on the N-terminus, or, in other cases, at a positive value, corresponding to a position at the C-terminus. However, spatial fluctuations of protons at room temperature are quite significant and lead to probability density distributions displaying a double-well character as shown 
by the presence of additional small peaks indicated by the arrows for each case (see also SI movie 2 and Suppl. Fig. S2 to observe proton fluctuations at $300 \mathrm{~K}$ ).

The preceding simulations treat the electrons quantum-mechanically but neglect quantum fluctuations of the nuclei. As indicated earlier, quantum effects in hydrogen bonded systems cause significant fluctuations of protons along H-bonds. Due to the large size of the $2 \mathrm{Y} 3 \mathrm{~J}$ and $2 \mathrm{Y} 3 \mathrm{~L}$ systems, ab initio simulations with quantum nuclei were only possible for the smallest system, 2Y2A, which already consists of over 400 atoms. In order to treat the electrons and nuclei quantum mechanically, one has to perform expensive path-integral molecular dynamics simulations (see Methods for details). Using 6 path-integral beads thus requires the simulation of over 2400 atoms for $2 \mathrm{Y} 2 \mathrm{~A}$. Note that the structural arrangement in 2Y2A permits two nitrogen atoms per chain to act as donors in the H-bond - one at the N-terminus and another at a lysine side chain, as highlighted in Fig. 3b. The corresponding probability density distributions of the proton transfer coordinates are shown in Fig. 3a, which compares classical- (black curves) and quantum-simulations (red curves). Consistent with previous studies, for which quantum effects have been shown to lead to a significant broadening of the O-H stretch in liquid water ${ }^{42}$, we similarly observe a significant delocalization of protons along the $\mathrm{H}$ bonds. This is evident from the non-zero probability density distribution of the proton transfer coordinate near 0 (red curve in Fig. 3a - see also SI movie 3 and Suppl. Fig. S3). We thus expect nuclear quantum fluctuations to lead to similar effects in $2 \mathrm{Y} 3 \mathrm{~J}$, since the H-bond lengths between the $\mathrm{N}$ - and C-termini are similar to those in 2Y2A $(\sim 2.7 \AA)$. In addition, 2Y3J features a double-well potential character in the classical simulations of the ground state. Due to the computational expense, we cannot repeat the quantum simulations for $2 \mathrm{Y} 3 \mathrm{~J}$ and hence we performed some benchmark simulations instead. We have included quantum effects at lower computational cost by thermostating high frequency modes in the system at a higher temperature, using the method described $\mathrm{in}^{43}$. As expected, and similar to $2 \mathrm{Y} 2 \mathrm{~A}$, the protons between the H-bonds of $2 \mathrm{Y} 3 \mathrm{~J}$ also exhibited delocalized character (see Suppl. Fig. S4).

\section{Proton transfer has a significant effect on the intrinsic fluorescence properties of protein fibrils}

In the previous section we used first principles simulations to show that proton transfer events can frequently occur between the $\mathrm{N}$-and $\mathrm{C}$-termini of protein fibrils and moreover, that proton delocalization is further enhanced by quantum fluctuations of protons. These phenomena are likely to have profound effects on the electronic energy states with associated changes in the optical properties of these materials. In order to explore how the fluorescence phenomenon observed in protein fibrils is coupled to proton transfer effects, we performed fluorescence spectroscopy experiments of protein fibrils either containing, or lacking aromatic amino acids and also of fibrils that were prepared in solutions with varying $\mathrm{pH}$ or in deuterium oxide $\left(\mathrm{D}_{2} \mathrm{O}\right)$. 
a)
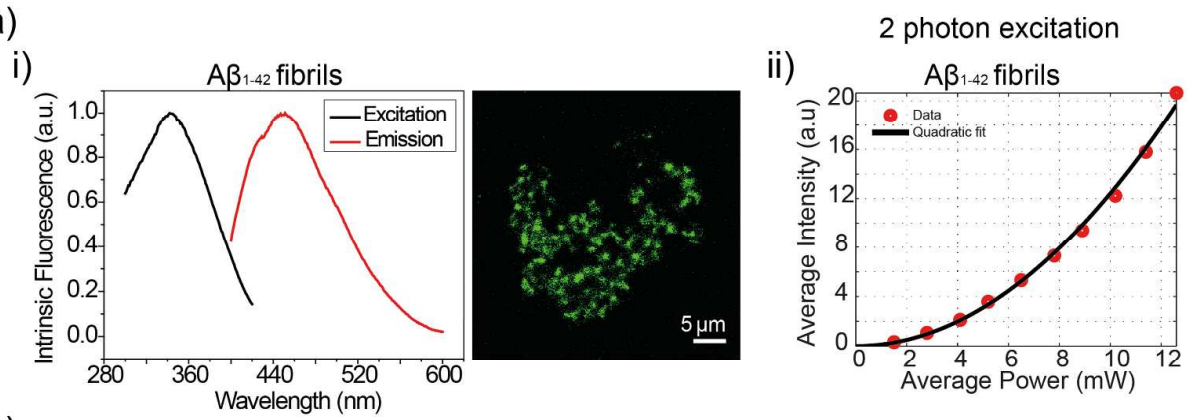

b)

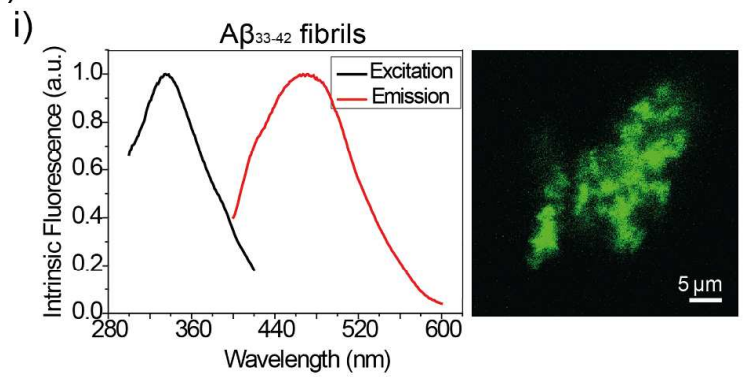

ii)

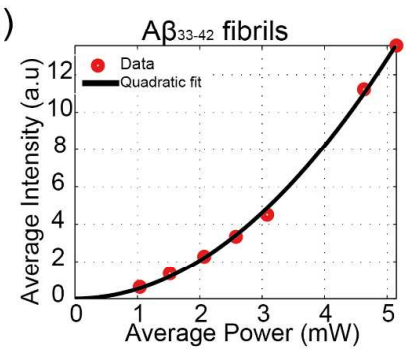

Figure 4: Protein fibrils display fluorophore-like properties even in the absence of aromatic residues. a) i) Excitation and emission spectra of the intrinsic fluorescence of $A B_{1-42}$ fibrils and corresponding fluorescence image of unlabelled aggregates obtained using a confocal microscope. ii) Two-photon fluorescence signals of $A B_{1-42}$ fibrils exhibit a quadratic dependence on the laser excitation power. b) i) Excitation and emission spectra of the intrinsic fluorescence of $A B_{33-42}$ fibrils and corresponding fluorescence image. ii) Two-photon fluorescence signals of $A B_{33-42}$ fibrils and a quadratic fit. For the excitation spectra the emission was centred at $450 \mathrm{~nm}$ and for the emission spectra the excitation was centred at $365 \mathrm{~nm}$. For the two-photon fluorescence excitation, the pulsed laser was set at $750 \mathrm{~nm}$ and the detection band was from 506 to 594 $n m$.

In particular, we studied the two structurally related amyloid- $\beta(A \beta)$ variants: $A \beta_{1-42}$ and $A \beta_{33-42}$, which both form typical $\beta$-sheet-rich fibrils ${ }^{44}$ but with the latter lacking any aromatic amino acids. We first show that both fibrils display very similar emission spectra upon near UV excitation at $365 \mathrm{~nm}$, with a peak emission wavelength occurring near $470 \mathrm{~nm}$ (Fig. 4a (i), b (i)). The spectral profiles were similar to those observed for many other fibrillar peptides and amyloid fibrils ${ }^{7}$ as well as for silk ${ }^{45}$. In an earlier study, multiphoton excitation was observed in amyloid fibrils and the phenomenon was interpreted in terms of the excitonic coupling between stacked aromatic residues present in the fibrils ${ }^{8}$. However, here we observed similar two-photon excited fluorescence from both aromatic residuecontaining $\left(\mathrm{A} \beta_{1-42}\right)$ and aromatic residue-free $\left(\mathrm{A} \beta_{33-42}\right)$ amyloid fibrils as shown in Fig. 4a (ii) and $\mathrm{b}$ (ii), respectively, and in Suppl. Fig. S9, which is proportional to the square of the excitation power ${ }^{46}$.

Our calculations discussed earlier show that proton transfer along H-bonds appears to be a prominent feature of $\beta$-sheet rich protein fibrils and this led us to reason that electronic energy states may be strongly affected by this effect. If this is the case, then we expect the intrinsic fluorescence to be sensitive to changes in solution $\mathrm{pH}$ due to the associated changes in protonation state of ionisable groups, especially near the N- and C-termini. In particular, in either fully protonated or deprotonated conditions both absorption and fluorescence properties should be significantly altered if proton transfer is indeed a driver of the fluorescence phenomenon. Under extreme acidic conditions $(\mathrm{pH}=0)$ 
all ionisable groups, including the $\mathrm{N}$ - and $\mathrm{C}$-termini, will be fully protonated and, conversely, these groups will be mainly deprotonated at $\mathrm{pH}=14$. We studied the fluorescence spectra of $A \beta_{1-42}$ fibrils formed in buffers with $\mathrm{pH}$ values ranging from $\mathrm{pH}=0$ to $\mathrm{pH}=14$ (see Methods section for experimental details). At the extreme conditions, i.e. $\mathrm{pH}=0$ and $\mathrm{pH}=14$, the optical properties of the fibrils are significantly perturbed compared to intermediate protonation states $(\mathrm{pH}=2$ and $\mathrm{pH}=7.4)$. In particular, for fibrils formed at $\mathrm{pH}=0$ and $\mathrm{pH}=14$ the fluorescence emission intensity is significantly decreased, the excitation peaks are blue-shifted and quenched in the lower energy range (340 to $400 \mathrm{~nm}$ ), as shown in Fig. 5a (i) and (ii).We also observe a higher emission intensity at $\mathrm{pH}=2$ as well as a redshifted excitation peak compared to $\mathrm{pH}=7.4$. These effects may be related to changes in proton transfer rates which affect the rate of non-radiative transitions and thereby the quantum yield. Similar observations were reported previously for other fluorophore systems containing proton donor and acceptor groups ${ }^{47}$, as well as for fluorescent proteins ${ }^{37}$.

a)

i)

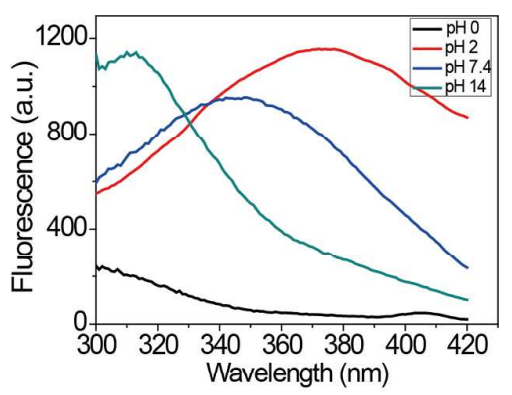

b)

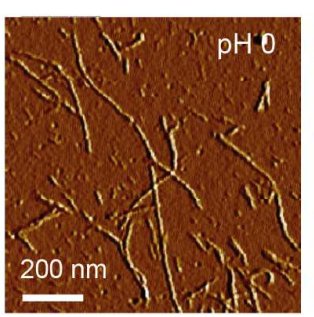

ii)
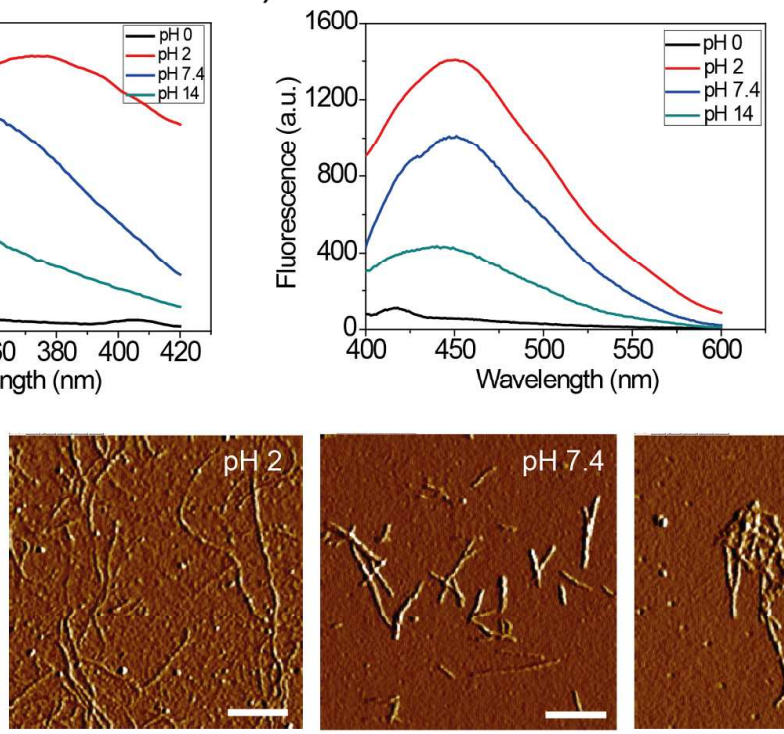

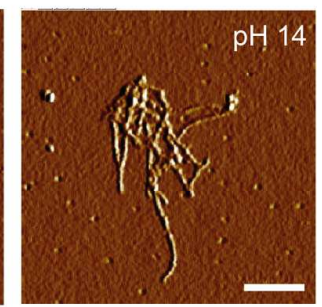

Figure 5: Proton transfer affects the fluorescence properties of protein fibrils. a) Excitation (i) and emission (ii) spectra of $A B_{1-42}$ fibrils in buffers of varying $\mathrm{pH}$ values. For the excitation spectra the emission window was centred at $450 \mathrm{~nm}$ and for emission spectra the excitation wavelength was centred at $365 \mathrm{~nm}$. b) AFM images showing the protein fibrils formed in buffers made in all the $\mathrm{pH}$ conditions for which the spectra were obtained in (a). The fibrillar nature of the protein was preserved for all buffer conditions investigated.

In order to probe further into the role of protons on optical properties of protein fibrils we substituted hydrogen atoms $(\mathrm{H})$ with deuterium $(\mathrm{D})$ and compared the ensuing emission spectra of $\mathrm{A} \beta_{1-42}$ fibrils formed in phosphate buffers (PBS, $\mathrm{pH}=7.4$ ) containing either $\mathrm{H}_{2} \mathrm{O}$ or $\mathrm{D}_{2} \mathrm{O}$ (Suppl. Fig. S10a). This substitution resulted in a blue shift of about $6 \mathrm{~nm}$ for the fluorescence emission peak in the deuterated fibrils, possibly pointing to nuclear effects (see later section). However, we were not able to discern changes in their excitation spectra. 
In order to test that the formation of fibrils was not affected by changes in solution $\mathrm{pH}$, for example via changes in electrostatic interactions between ionisable sites, or by $\mathrm{D}_{2} \mathrm{O}$ substitution we took AFM images of protein fibrils under all conditions (Fig. 5b and Suppl. Fig. S10b). Fibrils formed under all $\mathrm{pH}$ and isotope conditions tested and the $\mathrm{H}(\mathrm{D})$-bond network that stabilizes adjacent $\beta$-strands appeared to remain intact. Furthermore, fibril stability is not affected by the different protonation states at the $\mathrm{N}$ - and C-termini. It is worth noting that a previous computational study of amyloid forming peptides predicted that the formation of protein fibrils does not require the presence of charged $\mathrm{N}$ - and $\mathrm{C}$-termini. It is worth noting that a previous computational study of amyloid forming peptides predicted that the formation of protein fibrils does not require the presence of charged $\mathrm{N}$ - and C-termini ${ }^{48}$. The effect of $\mathrm{pH}$ on the intrinsic fluorescence of protein fibrils provides a link between the proton transfer observed in the MD simulations and the fluorescence phenomenon and supports the notion that the chromophore properties in such systems are strongly characterized by proton fluctuations within a network of H-bonds. Understanding exactly how the H-bond networks are affected by $\mathrm{pH}$ is an interesting question for future investigation using molecular dynamics simulations.

\section{Optical absorption spectra in model protein fibrils}

The ground-state simulations shown in Figs. 2 and 3 and SI movies 1 and 2 illustrate how proton transfer is facilitated in protein fibrils. To model the fluorescence phenomenon explicitly, similar calculations would have to be performed for the excited state, but this is currently computationally not feasible due to the size of the studied model systems. However, a wealth of information can be gained from an understanding of the molecular motions in the ground state and their effects on the absorption of light. Optical spectra were thus modelled with periodic time-dependent density functional theory (TD-DFT) using the Lioville-Lanczos method ${ }^{34}$, which is a suitable approach for large unit cells (ca. 700 atoms for $2 \mathrm{Y} 3 \mathrm{~J})$. 
a)

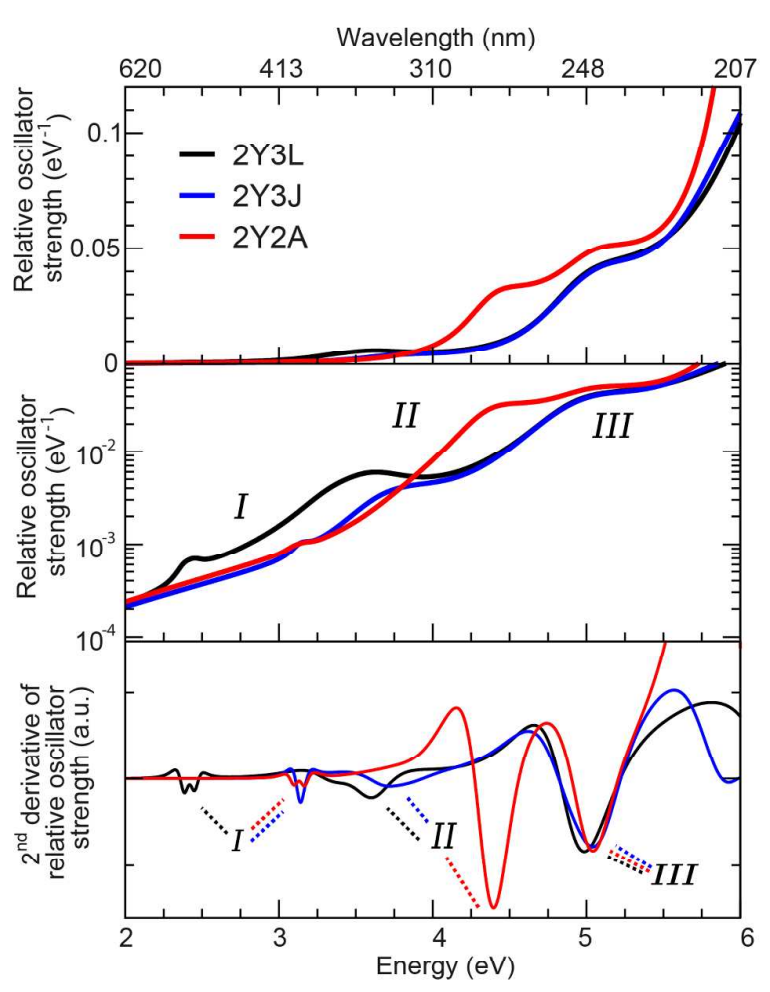

b)
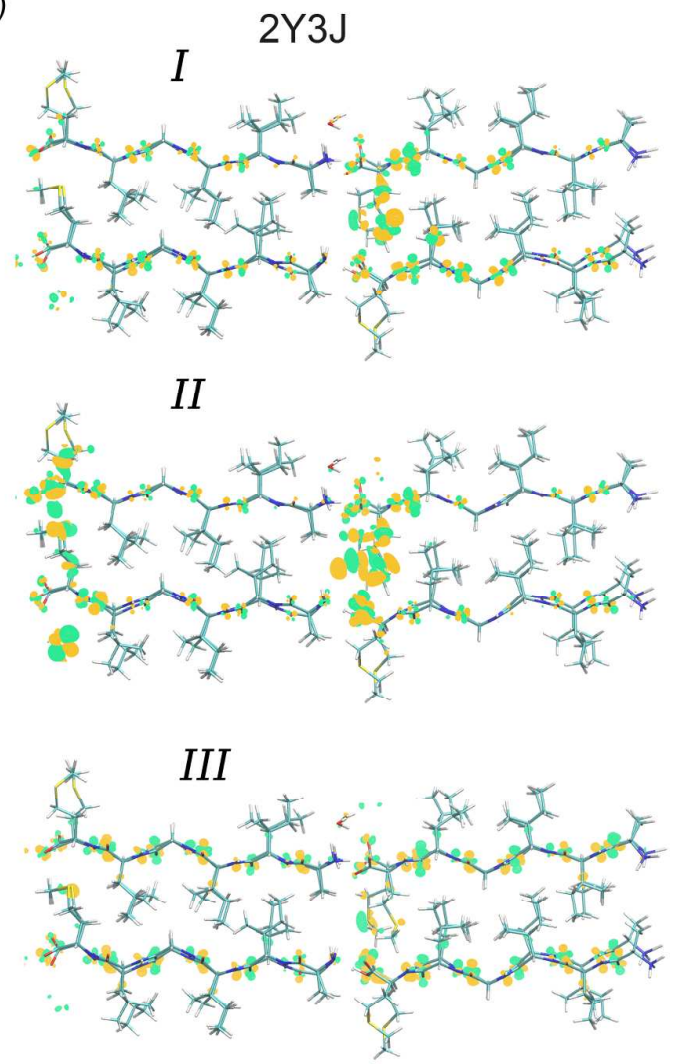

Figure 6: a) Computed absorption strength for the optimized at $0 \mathrm{~K}$ structures of $2 \mathrm{Y} 3 \mathrm{~J}$ (blue), $2 \mathrm{Y} 2 \mathrm{~A}$ (red) and $2 \mathrm{Y} 3 \mathrm{~L}$ (black), presented in linear (top panel) and logarithmic scales (middle panel). The intensity is expressed as oscillator strength normalized by the number of electrons in the unit cell to permit a comparison between the three systems. The bottom panel shows the second derivatives of the spectra (arbitrary units) which reveal the position of the absorption maxima (appearing as minima in the second derivative plot). b) The corresponding electronic densities for the three excitation peaks (denoted as I, II and III) are plotted for 2 Y3J. The iso-value for the response density is $3 E-4$ for I, 1.5E-3 for II and $2 E-3$ for III.

We computed the absorption spectra for the three structures 2Y3J, 2Y2A and 2Y3L at $0 \mathrm{~K}$ (Fig. 6a). In all three peptides we observe three peaks, one at $2.5-3 \mathrm{eV}(413-496 \mathrm{~nm})(\mathrm{I})$, another at $3.5-4 \mathrm{eV}$ $(310-350 \mathrm{~nm})$ (II) and finally at $5 \mathrm{eV}(\sim 246 \mathrm{~nm})$ (III). Note that only a qualitative comparison with experimental results can be made since model systems were used for the calculations. Also, the high degree of scattering prevailing in fibrillar material prevents the direct measurement of the absorption spectra. Instead fluorescence excitation scans were performed which mimic absorption spectra. In Fig. $6 \mathrm{~b}$ we show the excited state electron densities which give rise to each of the three maxima calculated for 2 Y3J. In all cases we observe significant electron delocalization in the excited state. We note furthermore that the low energy excitations (I and II) include electrons delocalized particularly in the vicinity of the N-C termini where, as discussed earlier, proton transfer is significant. They are a result of a mixing of $\pi$-conjugate states from the backbone with electronic states from residues ( $\mathrm{S}$ atoms). In contrast, for the highest excitation energy (III), the electron density is mainly localized along the peptide backbone and is insensitive to the environmental conditions since the corresponding absorption peaks are very similar for all three peptides suggestive of a typical $n-\pi^{*}$ transition. 
Moreover, analysis of the frontier orbitals is also coherent with the spatial distribution of the low lying excited state (see Suppl. Fig. S5 for 2Y3J). We do not currently find evidence for significant chargetransfer character in the excited states. However, this point will be explored specifically in future work in which the dynamics of the excited states will be taken into account.

We note that the apparent agreement between the positions of the first two peaks in the calculated absorption spectra and the experimentally observed excitation wavelengths has to be interpreted with caution because of the known sensitivity of the calculations to the choice of density functional used. A more detailed discussion on this point follows later in the article.

\section{Proton transfer events have a direct effect on absorption}

We then examined whether there is a coupling between the position of the proton along the proton transfer coordinate and the corresponding ground and excited state energies. Interestingly, we found that moving a single proton along the proton transfer coordinate in $2 \mathrm{Y} 3 \mathrm{~J}$ leads to a small but subtle spectral red shift of ca. $0.25 \mathrm{eV}(\sim 30 \mathrm{~nm})$ as seen in Fig. 7a. Note that whilst the energy of the ground state increases for proton movement along the transfer coordinate, there is a corresponding decrease in the excitation (and first excited state) energy (Fig. 7b). A similar type of coupling between the ground state potential and an excited charge-transfer state as a function of proton transfer has been shown by Domcke and co-workers for heterocyclic systems in the gas phase ${ }^{49}$.

a)

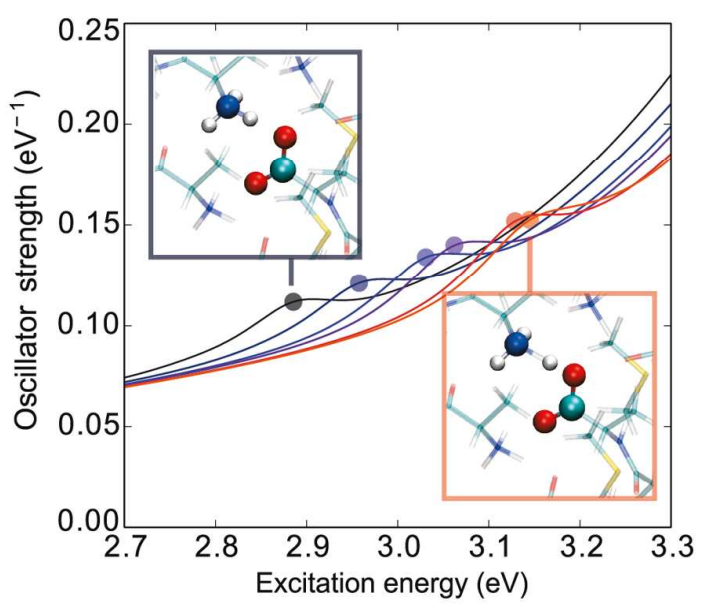

b)

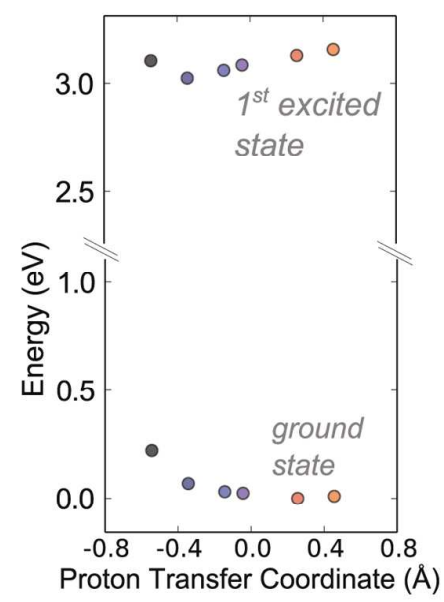

Figure 7: Proton transfer lowers the excitation energies. The figure demonstrates how movement of the proton between the $\mathrm{N}$ - and $\mathrm{C}$-terminus for one particular hydrogen bond in $2 \mathrm{Y} 3 \mathrm{~J}$ at $0 \mathrm{~K}$ can affect the excitation energy. a) Oscillator strength as a function of excitation energy and as a function of proton movement from the equilibrium position (inset framed in red) to the $\mathrm{N}$-terminus (inset framed in grey). Spectra corresponding to intermediate positions along the proton transfer coordinate are also shown. Proton movement results in a significant red shift in the excitation energy (from 390 to $430 \mathrm{~nm}$, i.e 3.2 to $2.9 \mathrm{eV}$ ). b) Ground state energies (bottom dots) and corresponding first excited state energies (top dots) as a function of the proton transfer coordinate, revealing energy minima located at different coordinates for the ground and the first excited states. 
We examined this behavior for several other $\mathrm{H}$-bond bridges between the $\mathrm{N}$ - and $\mathrm{C}$-termini in 2Y3J and observed similar trends. In addition to studying the effect of proton movement on the optical properties we looked at the effect of moving a proton between the $\mathrm{N}-\mathrm{H}$ and $\mathrm{C}=\mathrm{O}$ groups along the peptide backbone from different chains. The barrier for proton transfer in the ground-state in this case is significantly larger (by over $1 \mathrm{eV}$ ) than that for the $\mathrm{N}$ - and C-termini H-bonds and hence we do not observe any proton transfer events with these donor-acceptor groups even in our simulations at $300 \mathrm{~K}$. Indeed, recent ab initio simulations of model fibrils in the gas-phase had to increase the temperature to over $1000 \mathrm{~K}$ in order to observe proton transfer involving donor-acceptor groups of the peptide backbone ${ }^{50}$.

To evaluate the sensitivity of our results to the type of functional applied (BLYP versus CAMB3LYP), we performed benchmark calculations on a smaller model system, carved out of the full periodic $2 \mathrm{Y} 3 \mathrm{~J}$ system and consisting of $\sim 50$ atoms, as the full periodic system of $2 \mathrm{Y} 3 \mathrm{~J}$ is currently not computationally amendable to CAM-B3LYP. The model system consisted of one H-bond between the $\mathrm{N}$ - and C-termini and we compared the change of excitation energies upon proton movement, using both BLYP and CAM-B3LYP. It was found that both functionals yielded similar trends in changes of excitation energy along the proton transfer coordinate, however, the BLYP functional underestimated the absolute excitation energies compared to CAM-B3LYP by $\sim 2 \mathrm{eV}$ (see Suppl. Fig. S7). Hence, the apparent agreement between experimental and theoretically predicted absorption spectra must be interpreted with caution as mentioned earlier in the article. We note, however, that the smaller isolated cluster used cannot replicate environmental effects that may take place in the much larger periodic structure originally calculated using BLYP, and hence it is not possible to compare absolute magnitudes of energies calculated using the different functionals.

We assessed the effect of the three dimensional structure of protein fibrils on the magnitude of the excitation energies, and determined the absorption spectra for an artificially created system consisting of only two chains of 2Y3J. Stacking and backbone H-bonds do not occur in this system, except between the $\mathrm{N}$ - and C-termini of adjacent chains. We observe a blue shift of $\sim 0.5 \mathrm{eV}$ in the resulting spectra (Suppl. Fig. S8) thus supporting the notion that networks of H-bonds influence optical properties similar to our experimental observations shown in Suppl. Fig. S11b (ii).

\section{Absorption spectra can be further influenced by increasing temperature and by quantum effects}

The spectra shown earlier do not include the effects of thermal fluctuations. Thus, for $2 \mathrm{Y} 3 \mathrm{~J}$ we also computed the optical spectra obtained by averaging over configurations sampled from simulations at $300 \mathrm{~K}$ (Fig. 8a). As a visual guide, the vertical lines in the figure correspond to the positions of the lowest peaks of excitation energies obtained at $0 \mathrm{~K}$. The inclusion of temperature results in a broader distribution and an increased oscillator strength at lower excitation energies. Extracting correlations 
between these energies and the motion of specific protons is challenging due to the many different degrees of freedom within the system. However, in order to test the robustness of our results we have repeated the above calculations for two more H-bonds and find similar features.

Recent simulations of protonated water have revealed that nuclear quantum effects broaden the electronic density of states and hence introduce electronic levels in both the ground and excited state that are classically inaccessible ${ }^{23}$. We have shown earlier, that for $2 \mathrm{Y} 2 \mathrm{~A}$ quantum fluctuations of the protons lead to significant changes in the proton delocalization along H-bonds in the ground state. Furthermore, previous theoretical studies have shown that quantum effects can lead to a significant broadening of absorption spectra ${ }^{25,51,52}$. Since nuclear quantum effects affect the properties of protons the most, an effect on the absorption spectra would once again strongly implicate the role of proton delocalization on the optical properties.

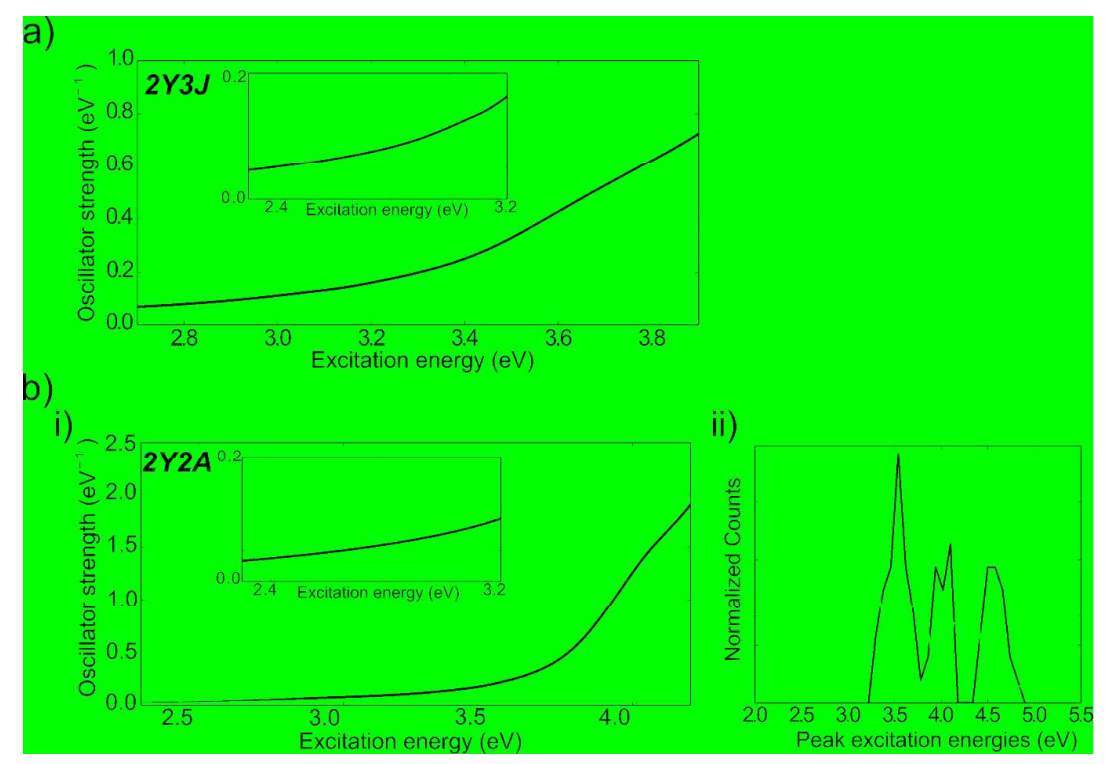

Figure 8: Absorption is enhanced by thermal fluctuations and nuclear quantum effects. a) Oscillator strength computed for 2Y3J: average spectra (black thick line) and its frequency-dependent standard deviation (shaded grey area). Scattered points correspond to the maxima calculated for all the spectra obtained at $300 \mathrm{~K}$, while vertical lines correspond to maxima of the $0 \mathrm{~K}$ structure. The inset shows a zoomed-in low-frequency region. b) i) Oscillator strength computed for 2Y2A: (classical nuclei: black line, quantum nuclei: red line) and its frequency-dependent standard deviation (shaded grey and red area for classical and quantum nuclei, respectively). Black and red scattered points correspond to the maxima calculated for all the spectra, while the vertical line corresponds to the maximum of the $0 \mathrm{~K}$ structure. The inset shows a zoomed-in lowfrequency region. For both $2 Y_{3} \mathrm{~J}$ and $2 \mathrm{Y} 2 \mathrm{~A}$ these values were evaluated over frames extracted along a room temperature ab initio molecular dynamics simulation. ii) Corresponding distribution of calculated peak excitation energies for $2 Y 2 \mathrm{~A}$ (classical nuclei: black line, quantum nuclei: red line). The figure displays three peaks that correspond to the three excited states. The quantum nuclei simulations show a red shift of the excited states. Each excited state distribution is normalized such that the integrated area is equal to one.

We thus computed optical spectra via sampling configurations obtained from path-integral simulations of 2Y2A (see Methods for details). The theory behind this methodology has been described in detail by Marx and co-workers and was successfully applied for various soft-mode systems $^{25,51-53}$. The method relies on the assumption that the Born-Oppenheimer approximation holds 
true and hence any relaxation of the environment during excitation is neglected. The oscillator strength of the optical absorption for $2 \mathrm{Y} 2 \mathrm{~A}$ was thus determined for both classical and quantum simulations. Fig. $8 \mathrm{~b}$ shows the average spectra obtained from classical (black) and quantum simulations (red). Interestingly, if we focus on the region of the plot on the left of the vertical line (the excitation peak at $0 \mathrm{~K}$ for $2 \mathrm{Y} 2 \mathrm{~A}$ ), we observe that the quantum simulations show a significant nonzero optical response. This is more clearly seen in Fig. $8 \mathrm{~b}$ (ii) which shows a histogram of the excitation energies. Another way to interpret this is that nuclear quantum effects result in a red-shift of the excitation energies relative to those observed classically. Besides this difference, the quantum simulations also feature larger variation in the magnitudes of the oscillator strength over the entire energy range examined. These results show that quantum effects, which alter the fluctuations of protons along the $\mathrm{H}$-bonds, have an additional impact on the absorption spectra.

\section{Hydrogen bond-rich protein fibrils form a non-conventional structural chromophore}

Our results up to this point provide experimental and theoretical support of a structure-specific fluorophore that is formed in protein fibrils and in structurally similar protein assemblies, rich in $\mathrm{H}-$ bonds. The associated fluorescence phenomenon appears to be strongly coupled to proton transfer events taking place in the interconnected network of H-bonds characteristic of such structures. An examination of the excited state electron density at $0 \mathrm{~K}$, as shown for $2 \mathrm{Y} 3 \mathrm{~J}$ in Fig. $6 \mathrm{~b}$, reveals that it is delocalized and involves the $\mathrm{N}$ - and $\mathrm{C}$-termini as well as the surrounding amino acids and thus spans both along the fibril axis and across protofibrils (i.e. stacks of $\beta$-strands). Similarly, for 2Y2A and 2Y3L we observe that low-lying transitions involve more than one chain and that the corresponding electron density is delocalized over more than one strand.

a)

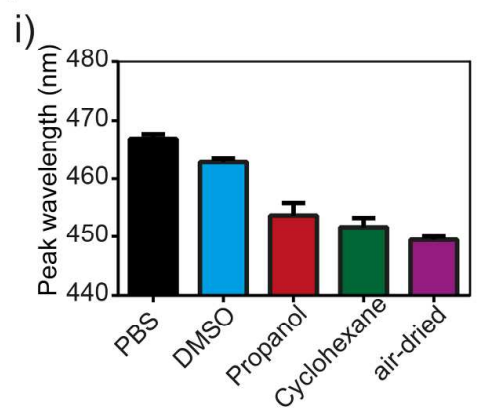

ii)

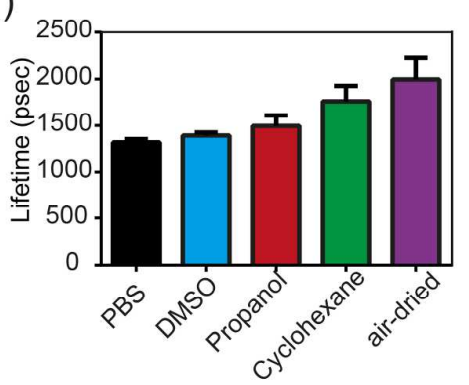

b)

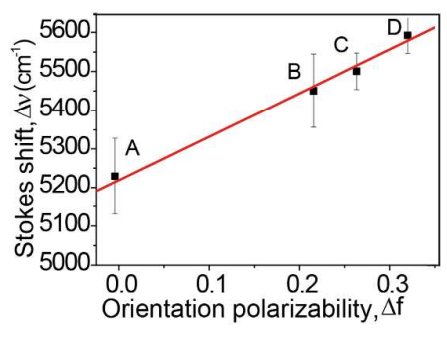

Figure 9: a) i) Peak emission wavelength and ii) fluorescence lifetime of the intrinsic fluorescence of $A B_{1-42}$ fibrils in different solvents and in their fully dried state. b) Lippert plot displaying the Stokes shifts of the fluorescence of $A B_{1-42}$ fibrils in wavenumbers against the orientational polarizability $\Delta f$ of different solvents. The letters refer to A: Cyclohexane, B: Butoxyethanol, C: DMSO and D: PBS. The dipole moment difference $\triangle \mu$ between ground and excited state is given by the slope of the plot.

The theoretical predictions point to a non-conventional delocalized structural chromophore spreading over multiple strands which is modulated by proton transfer. In order to ascertain this hypothesis we 
performed experiments to get an estimate of the change in dipole moment $(\Delta \mu)$ that occurs upon excitation of this protein fibril fluorophore. We measured the fluorescence Stokes shifts $(\Delta v)$ for protein fibrils suspended in solvents of varying polarity and extracted the dipole moment changes from a Lippert plot ${ }^{16,54}$. We found that there is a significant (up to $20 \mathrm{~nm}$ ) Stokes shift change by going from polar to non-polar solvents (Fig. 9a (i)), which is similar to values measured for conventional fluorophores ${ }^{55,56}$. In addition, we also measured the concomitant changes in the fluorescence lifetime and observed a strong inverse correlation with solvent polarity for $A \beta_{1-42}$ fibrils (Fig. 9a (ii)). The same effect was observed in $A \beta_{33-42}$ fibrils (Suppl. Fig. S11a). Both these observations point to an excitation involving a change in the charge distribution of the fluorophore.

In order to estimate the dipole moment changes and thus the extent of charge re-arrangement in the fluorophore associated with the measured Stokes shifts, we used the Lippert-Mataga equation ${ }^{54}$ (see SI). In order to use the Lippert-Mataga equation for the calculation of the dipole moment changes, several assumptions were made. In particular, only effects caused by the solvent polarity to the fluorophore were considered, whereas any excited state or charge interactions and any solvent specific effects were excluded and thus the environment was treated as a dielectric continuum. Furthermore, we assumed that the Onsager cavity was spherical and thus similar to common fluorophores. It is worth noting first that the magnitudes of the Stokes shifts obtained here in polar solvents are quite large compared to those observed for aromatic residues such as tryptophan ${ }^{57}$. Furthermore, our calculations suggest that the excitation involves several protein fibril chains (Fig. 6b). We thus used the size between two adjacent chains ( $\beta$-strands) in the protofibril as the reference value for the Onsager radius. Upon changing the size of the Onsager cavity from 2 to 4 , or to $6 \beta$-strands respectively, which corresponds to a change in the cavity radius from 4 to $23 \AA^{11}$ we get $\Delta \mu$ values of 0.3 Debye units (D), $1.05 \mathrm{D}$, and $3 \mathrm{D}$, respectively (see SI). Interestingly, all protein fibrils studied displayed similar values (see Suppl. Fig. S11).

Despite these assumptions, the sensitivity of the optical properties of fibrils to solvent polarity indicates that the structural fluorophore displays a large polarity. The results suggest that charge redistribution takes place in the excited state and that an Onsager cavity radius equivalent to more than $4 \beta$-strands is required for $\Delta \mu$ values to be higher than $1 \mathrm{D}$. Similar values for $\Delta \mu$ were found in corresponding experiments performed with $\mathrm{A} \beta_{33-42}$ fibrils (see SI).

We have previously shown that amyloid proteins in their monomeric form do not display any fluorescence ${ }^{7}$. However, since the $A \beta_{1-42}$ peptide aggregates spontaneously, we used the monomeric form of another protein, K18Tau, which remains monomeric while dissolved in the different solvents. This protein similarly forms $\beta$-sheet rich fibrils but only upon exposure to heparin ${ }^{58}$. Our data provide evidence that K18Tau does not display any fluorescence in its monomeric, disordered form in all different solvents studied. On the other hand, the fluorescence properties and the analysis of the dipole 
moment change of its fibrillar form are similar to those of $A \beta_{1-42}$ and $A \beta_{33-42}$ fibrils (Suppl. Fig. S11b). This confirms that the three-dimensional structure attained by the protein fibrils, including the formation of protofilaments which bring the $\mathrm{N}$ - and C-termini in close proximity, as well as their highly ordered network of H-bonds are indeed essential for the emergence of the fluorescence.

\section{Conclusions:}

In this work we have combined state-of-the-art ab initio molecular dynamics simulations and fluorescence spectroscopy in order to elucidate the origin of the anomalous fluorescence phenomena observed in H-bond-rich protein fibrils. The experimental results demonstrate that, unlike conventional fluorophores, fluorescence in these fibrils does not require the presence of aromatic residues or more generally, conjugated $\pi$-electron systems. Our simulations for the electronic ground state show that protons situated along strong H-bonds involving $\mathrm{N}-\mathrm{C}$ termini can be exceptionally labile. This leads to situations for which the proton can be stabilized on either the $\mathrm{N}$ - or the Cterminus, which ultimately results in the proton transfer coordinate potential having a double well character (Fig. 2c). Furthermore, due to the quantum mechanical nature of the proton, nuclear quantum fluctuations along the H-bonds lead to proton delocalization and hence to significant broadening of the proton transfer distributions (Fig. 3a). These fundamental features that characterize the structure of amyloid protein fibrils have, to the best of our knowledge, not been reported before in the context of hydrogen bonding in fibrils and this is likely to have an impact on several physicochemical properties and the observed optical properties of these systems.

In Fig. 10 we illustrate two conceptual scenarios on how the shape of different ground and excited state potentials can change the fate of potential fluorescence emission. In Fig. 10a the ground and excited states are shown for a situation for which the ground state is a single well potential and thus proton transfer incurs a huge activation barrier. As shown in several publications on heterocyclic molecules ${ }^{20-22}$, a common deactivating path involving the proton transfer coordinate is based on the occurrence of a so-called conical intersection between the excited and the ground state along the same coordinate. Fig. 10b illustrates the coupling between a double-well ground state potential and the excited state. Comparing Fig. 10a to $10 \mathrm{~b}$ we observe that since the electronic excitation energies are much larger than thermal energies associated with the proton fluctuations in the ground state, conical intersections are avoided and lead to situations for which the system is more likely to be trapped in the excited state leading to fluorescence. It should be mentioned that the scenarios depicted in Fig. 10 and their potential implication to fluorescence solely depend on the stiffness of the proton potential in the ground-state, and are thus less sensitive to computational approximations such as the choice of the density functional. The possibility of finding a conical intersection between the ground and excited state proton potential will be reduced if it has a double-well character and/or has become asymmetric due to nuclear quantum fluctuations. 
a)

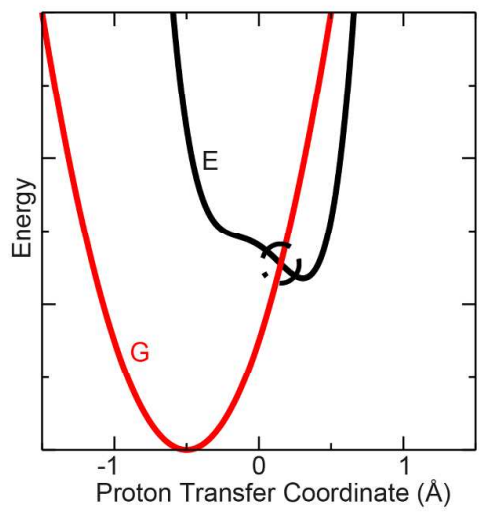

b)

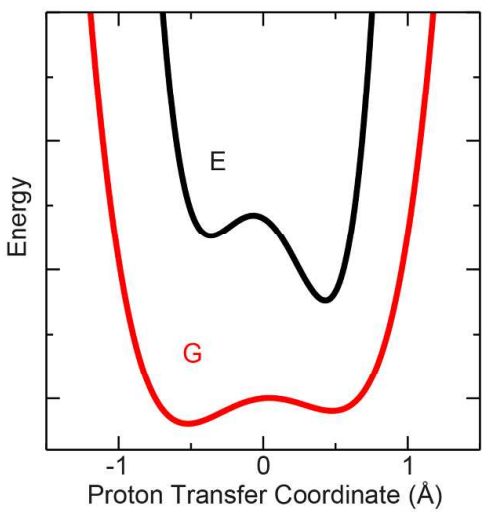

Figure 10: A cartoon sketch for the proton motion within a double-well potential which can alter the coupling between the ground and excited state potentials. Here, we focus on the lowest excited state as we assume from Kasha's rule that this will be the most dominant state from which fluorescence will occur. a) The ground-state potential is harmonic and proton transfer incurs a huge activation barrier. The ground-state potential forms a conical intersection with the excited state which could thus lead to deactivation and inhibit fluorescence. $b$ ) The presence of a double-well potential caused by proton transfer along a $\mathrm{H}$-bond between the $\mathrm{N}$ - and $\mathrm{C}$-termini in the case of the fibrils prevents the formation of a conical intersection.

It should also be stressed that the scenarios depicted in Fig. 10 do not include features such as the reorganization of the solvent or of the structural environment in response to excitation. While these could certainly be included and would probably play a role during the relaxation process that precede emission, this does not change our qualitative conclusions. Future work is required using both theoretical and experimental approaches to understand the complex physical chemistry of these systems.

In summary, we have demonstrated using ground-state $a b$ initio molecular dynamics simulations that specific H-bonds in protein fibrils are permissive to proton transfer, leading to situations for which the proton can be found on either the $\mathrm{N}$ - or the $\mathrm{C}$-terminus and thus to the creation of a double well ground-state potential that is likely to have a consequence on both the absorption and emission characteristics of the system. We have calculated absorption spectra explicitly, and find that proton transfer lowers the excitation energy levels, possibly shifting them into the near UV or visible range, as observed experimentally. The presented ab initio simulations together with our experimental data reveal a significant effect of $\mathrm{pH}$, isotope, and fibril structure on fibril absorption and/or fluorescence properties, and they suggest a significant role of proton transfer processes on spectral properties of fibrillar protein structures. The presence of a structure-specific fluorophore in these structures may have a wide range of implications in biological systems, such as for electrochemical functions and signal propagation. Since amyloid proteins, which form such fibrils, are at the heart of neurodegenerative and other aggregation-related diseases, this fluorescence phenomenon could be used as a basis for assays of protein aggregation, both in basic research, e.g. as a label-free readout of protein amyloidogenesis ${ }^{5,7,59}$, and for drug discovery, in the application of fluorescence based assays for the development of aggregation-inhibiting compounds. Finally, their photonics and potential 
semiconductor properties may make these structures suitable for applications in biosensing and provide the basis for potential future technologies, for example, for a new class of bio-inspired, selfassembled optical materials towards the development of biomolecular photonics devices.

\section{Acknowledgements:}

We thank Prof. M. Sauer for useful discussions. This work was funded by grants from the Medical Research Council UK (MR/K015850/1 and MR/K02292X/1), Alzheimer Research UK (ARUKEG2012A-1), U.K. EPSRC (EP/H018301/1) and the Welcome Trust (089703/Z/09/Z). D.P. wishes to acknowledge support from the Swiss National Science Foundation and the Welcome Trust through personal fellowships. We thank LMB Visual Aids for graphics support.

\section{Supporting Information:}

Additional details regarding: optimization at $0 \mathrm{~K}$, molecular dynamics at $300 \mathrm{~K}$, excited state calculations and orbitals, comparison between BLYP and CAM-B3LYP, 2 photon excitation and Lippert plots and analysis. Figures S1-S10. This information is available free of charge via the internet at http://pubs.acs.org.

\section{References:}

(1) Shukla, A.; Mukherjee, S.; Sharma, S.; Agrawal, V.; Radha Kishan, K. V; Guptasarma, P. Arch. Biochem. Biophys. 2004, 428, 144-53.

(2) Sharpe, S.; Simonetti, K.; Yau, J.; Walsh, P. Biomacromolecules 2011, 12, 1546-55.

(3) Tcherkasskaya, O. Protein Sci. 2007, 16, 561-71.

(4) Amdursky, N.; Molotskii, M.; Aronov, D.; Adler-Abramovich, L.; Gazit, E.; Rosenman, G. Nano Lett. 2009, 9, 3111-5.

(5) Kaminski Schierle, G. S.; Bertoncini, C. W.; Chan, F. T. S.; van der Goot, A. T.; Schwedler, S.; Skepper, J.; Schlachter, S.; van Ham, T.; Esposito, A.; Kumita, J. R.; Nollen, E. A. A.; Dobson, C. M.; Kaminski, C. F. Chemphyschem 2011, 12, 673-80.

(6) Chan, F. T. S.; Pinotsi, D.; Kaminski Schierle, G.; Kaminski, C. F. In Bio-nanoimaging Protein Misfolding \& Aggregation; Uversky, V. N.; Lyubchenko, Y. L., Eds.; Academic Press: Waltham, MA, USA, 2014.

(7) Pinotsi, D.; Buell, A. K.; Dobson, C. M.; Kaminski Schierle, G. S.; Kaminski, C. F. Chembiochem 2013, 14, 846-50.

(8) Hanczyc, P.; Samoc, M.; Norden, B. Nat. Photonics 2013, 7, 969-972.

(9) Del Mercato, L. L.; Pompa, P. P.; Maruccio, G.; Della Torre, A.; Sabella, S.; Tamburro, A. M.; Cingolani, R.; Rinaldi, R. Proc. Natl. Acad. Sci. U. S. A. 2007, 104, 18019-24.

(10) Nelson, R.; Sawaya, M. R.; Balbirnie, M.; Madsen, A. Ø.; Riekel, C.; Grothe, R.; Eisenberg, D. Nature 2005, 435, 773-8.

(11) Fitzpatrick, A. W. P.; Debelouchina, G. T.; Bayro, M. J.; Clare, D. K.; Caporini, M. A.; Bajaj, V. S.; Jaroniec, C. P.; Wang, L.; Ladizhansky, V.; Müller, S. A.; MacPhee, C. E.; Waudby, C. A.; Mott, H. R.; De Simone, A.; Knowles, T. P. J.; Saibil, H. R.; Vendruscolo, M.; Orlova, E. 
V; Griffin, R. G.; Dobson, C. M. Proc. Natl. Acad. Sci. U. S. A. 2013, 110, 5468-73.

Baldwin, A. J.; Knowles, T. P. J.; Tartaglia, G. G.; Fitzpatrick, A. W.; Devlin, G. L.;

Shammas, S. L.; Waudby, C. A.; Mossuto, M. F.; Meehan, S.; Gras, S. L.; Christodoulou, J.;

Anthony-Cahill, S. J.; Barker, P. D.; Vendruscolo, M.; Dobson, C. M. J. Am. Chem. Soc. 2011, $133,14160-3$.

(13) Chan, F. T. S.; Kaminski Schierle, G. S.; Kumita, J. R.; Bertoncini, C. W.; Dobson, C. M.; Kaminski, C. F. Analyst 2013, 138, 2156-62.

(14) Kuhn, H. J. Chem. Phys. 1949, 17, 1198-1212.

(15) Lagorio, M. G.; Cordon, G. B.; Iriel, A. Photochem. Photobiol. Sci. 2015, 14, 1531-1537.

(16) Lakowicz, J. R. Principles of Fluorescence Spectroscopy | 3rd edition; Springer US: New York, 2006.

(17) Schauenstein, E.; Perko, G. Zeitschrift für Elektrochemie, Berichte der Bunsengesellschaft für Phys. Chemie 1953, 57, 927-934.

(18) Arnold, W. D.; Oldfield, E. J. Am. Chem. Soc. 2000, 122, 12835-12841.

(19) Dingley, A. J.; Grzesiek, S. J. Am. Chem. Soc. 1998, 120, 8293-8297.

(20) Domcke, W.; Yarkony, D. R.; Köppel, H. Conical Intersections (World Scientific) http://www.worldscientific.com/worldscibooks/10.1142/7803.

(21) Sobolewski, A. L.; Domcke, W. Chem. Phys. Lett. 1999, 315, 293-298.

(22) Sobolewski, A. L.; Domcke, W.; Dedonder-Lardeux, C.; Jouvet, C. Phys. Chem. Chem. Phys. 2002, 4, 1093-1100.

(23) Giberti, F.; Hassanali, A. A.; Ceriotti, M.; Parrinello, M. J. Phys. Chem. B 2014, 118, 1322635 .

(24) Wang, L.; Fried, S. D.; Boxer, S. G.; Markland, T. E. Proc. Natl. Acad. Sci. U. S. A. 2014, 111, 18454-18459.

(25) Della Sala, F.; Rousseau, R.; Görling, A.; Marx, D. Phys. Rev. Lett. 2004, 92, 183401-4.

(26) VandeVondele, J.; Krack, M.; Mohamed, F.; Parrinello, M.; Chassaing, T.; Hutter, J. Comput. Phys. Commun. 2005, 167, 103-128.

(27) Lee, C.; Yang, W.; Parr, R. G. Phys. Rev. 1988, B37, 785-789.

(28) Goedecker, S.; Teter, M.; Hutter, J. Phys. Rev. 1996, B54, 1703-1710.

(29) Grimme, S.; Antony, J.; Ehrlich, S.; Krieg, H. J. Chem. Phys. 2010, 132, 1-19.

(30) Byrd, R. H.; Lu, P.; Nocedal, J.; Zhu, C. SIAM J. Sci. Comput. 1995, 16, 1190-1208.

(31) Bussi, G.; Donadio, D.; Parrinello, M. J. Chem. Phys. 2007, 126, 1-7.

(32) Ceriotti, M.; Manolopoulos, D. E. Phys. Rev. Lett. 2012, 109, 100604.

(33) Giannozzi, P.; Baroni, S.; Bonini, N.; Calandra, M.; Car, R.; Cavazzoni, C.; Ceresoli, D.; Chiarotti, G. L.; Cococcioni, M.; Dabo, I.; Dal Corso, A.; de Gironcoli, S.; Fabris, S.; Fratesi, G.; Gebauer, R.; Gerstmann, U.; Gougoussis, C.; Kokalj, A.; Lazzeri, M.; Martin-Samos, L.; Marzari, N.; Mauri, F.; Mazzarello, R.; Paolini, S.; Pasquarello, A.; Paulatto, L.; Sbraccia, C.; Scandolo, S.; Sclauzero, G.; Seitsonen, A. P.; Smogunov, A.; Umari, P.; Wentzcovitch, R. M. J. Phys. Condens. Matter 2009, 21, 395502 (19pp).

(34) Malcığlu, O. B.; Gebauer, R.; Rocca, D.; Baroni, S. Comput. Phys. Commun. 2011, 182, 1744-1754.

(35) Teplow, D. B. Methods Enzymol. 2006, 413, 20-33.

(36) Pérez, A.; Tuckerman, M. E.; Hjalmarson, H. P.; von Lilienfeld, O. A. J. Am. Chem. Soc. 2010, 132, 11510-5. 
(37) Oltrogge, L. M.; Wang, Q.; Boxer, S. G. Biochemistry 2014, 53, 5947-57.

(38) Hutter, J.; Iannuzzi, M.; Schiffmann, F.; VandeVondele, J. Wiley Interdiscip. Rev. Comput. Mol. Sci. 2014, 4, 15-25.

(39) Colletier, J.-P.; Laganowsky, A.; Landau, M.; Zhao, M.; Soriaga, A. B.; Goldschmidt, L.; Flot, D.; Cascio, D.; Sawaya, M. R.; Eisenberg, D. Proc. Natl. Acad. Sci. U. S. A. 2011, 108, 16938-43.

(40) Guidon, M.; Hutter, J.; VandeVondele, J. J. Chem. Theory Comput. 2009, 5, 3010-3021.

(41) Guidon, M.; Hutter, J.; VandeVondele, J. J. Chem. Theory Comput. 2010, 6, 2348-2364.

(42) Morrone, J. A.; Car, R. Phys. Rev. Lett. 2008, 101, 017801-4.

(43) Ceriotti, M.; Bussi, G.; Parrinello, M. J. Chem. Theory Comput. 2010, 6, 1170-1180.

(44) Li, H.; Monien, B. H.; Lomakin, A.; Zemel, R.; Fradinger, E. A.; Tan, M.; Spring, S. M.; Urbanc, B.; Xie, C.-W.; Benedek, G. B.; Bitan, G. Biochemistry 2010, 49, 6358-64.

(45) Georgakoudi, I.; Tsai, I.; Greiner, C.; Wong, C.; DeFelice, J.; Kaplan, D. Opt. Express 2007, $15,1043$.

(46) Xu, C.; Webb, W. W. J. Opt. Soc. Am. B 1996, 13, 481.

(47) Stryer, L. J. Am. Chem. Soc. 1966, 88, 5708-5712.

(48) Baftizadeh, F.; Biarnes, X.; Pietrucci, F.; Affinito, F.; Laio, A. J. Am. Chem. Soc. 2012, 134, 3886-94.

(49) Sobolewski, A. L.; Domcke, W. Chemphyschem 2006, 7, 561-4.

(50) Porter, D.; Vollrath, F. Soft Matter 2013, 9, 643-646.

(51) Svoboda, O.; Ončák, M.; Slavíček, P. J. Chem. Phys. 2011, 135, 154301-16.

(52) Svoboda, O.; Ončák, M.; Slavíček, P. J. Chem. Phys. 2011, 135, 154302-16.

(53) Law, Y. K.; Hassanali, A. A. J. Phys. Chem. A 2015, 119, 10816-27.

(54) Lippert, E. Berichte der Bunsengesellschaft für Phys. Chemie 1957, 61, 962-975.

(55) Singh, M. K.; Pal, H.; Bhasikuttan, A. C.; Sapre, A. V. Photochem. Photobiol. 1998, 68, $32-$ 38.

(56) Seliskar, C. J.; Brand, L. J. Am. Chem. Soc. 1971, 93, 5414-5420.

(57) Pal, S. K.; Peon, J.; Bagchi, B.; Zewail, A. H. J. Phys. Chem. B 2002, 106, 12376-12395.

(58) Goedert, M.; Jakes, R.; Spillantini, M. G.; Hasegawa, M.; Smith, M. J.; Crowther, R. A. Nature 1996, 383, 550-3.

(59) Michel, C. H.; Kumar, S.; Pinotsi, D.; Tunnacliffe, A.; St George-Hyslop, P.; Mandelkow, E.; Mandelkow, E.-M.; Kaminski, C. F.; Kaminski Schierle, G. S. J. Biol. Chem. 2014, 289, $956-$ 67. 


\section{TOC figure:}

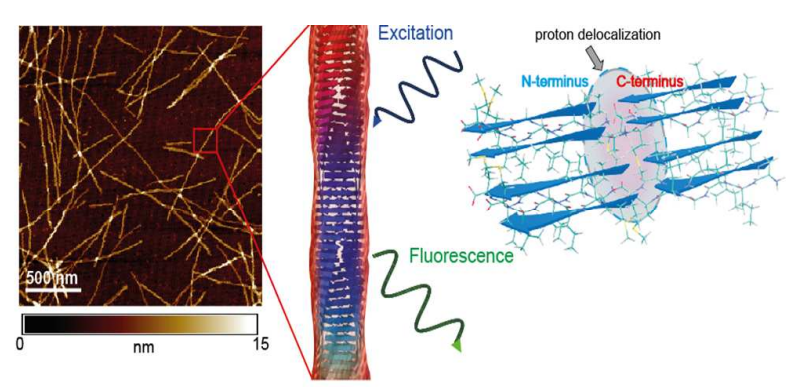


a)

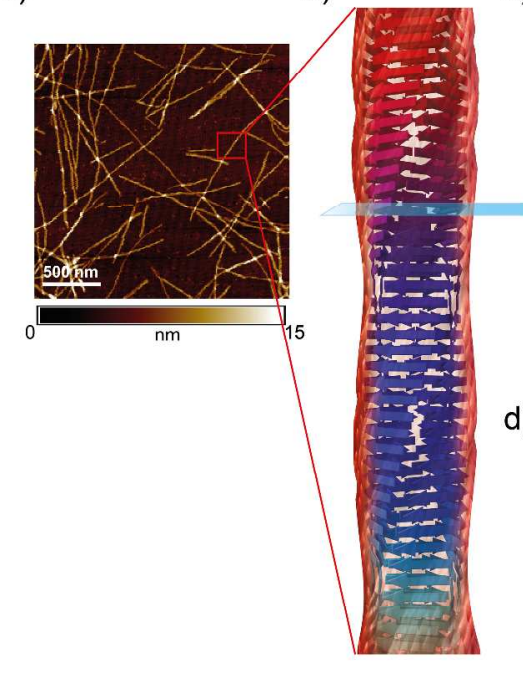

c)

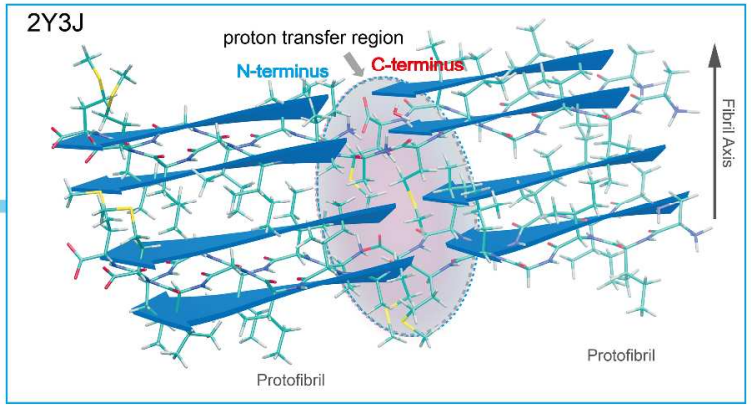

d)

i) $2 Y 3 \mathrm{~L}$

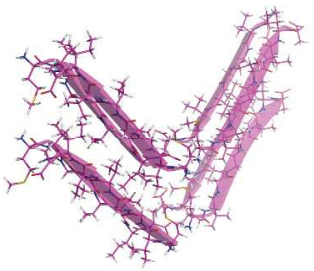

$510 \times 313 \mathrm{~mm}(300 \times 300$ DPI $)$ ii) $2 \mathrm{Y} 2 \mathrm{~A}$

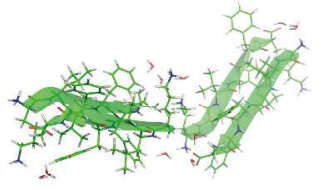


a)

i)

ii)

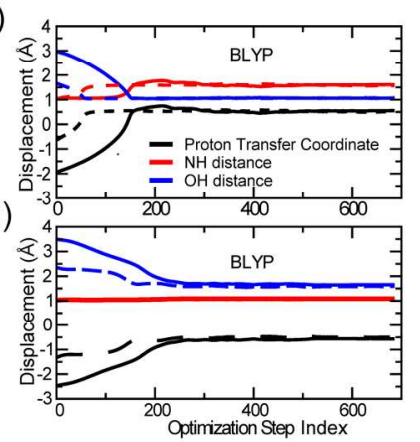

b)

i)

ii)

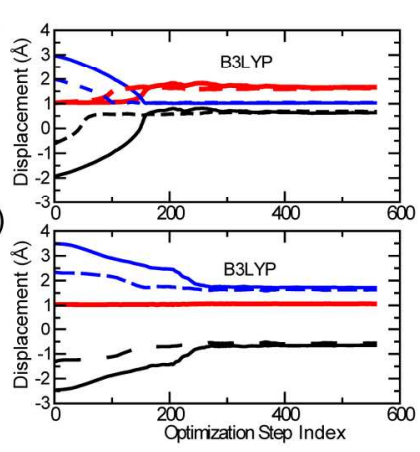

c)

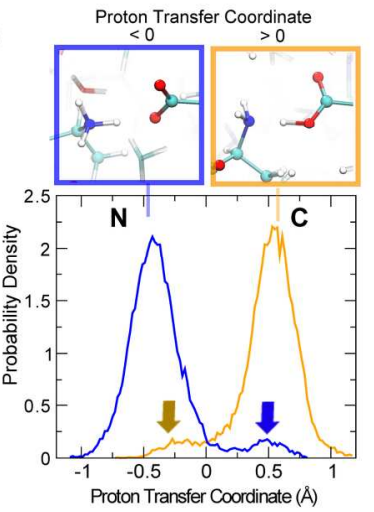

$90 \times 36 \mathrm{~mm}(600 \times 600 \mathrm{DPI})$ 
a)

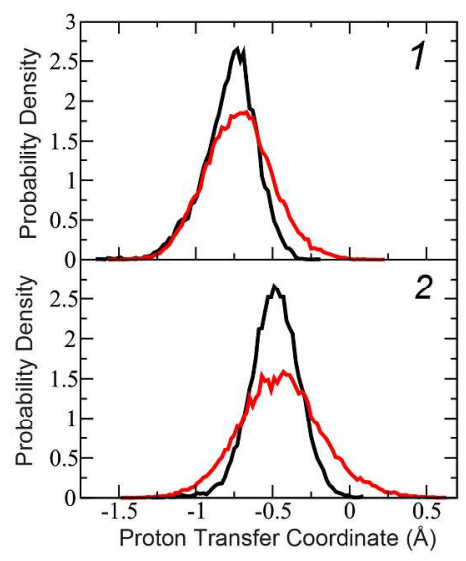

b)

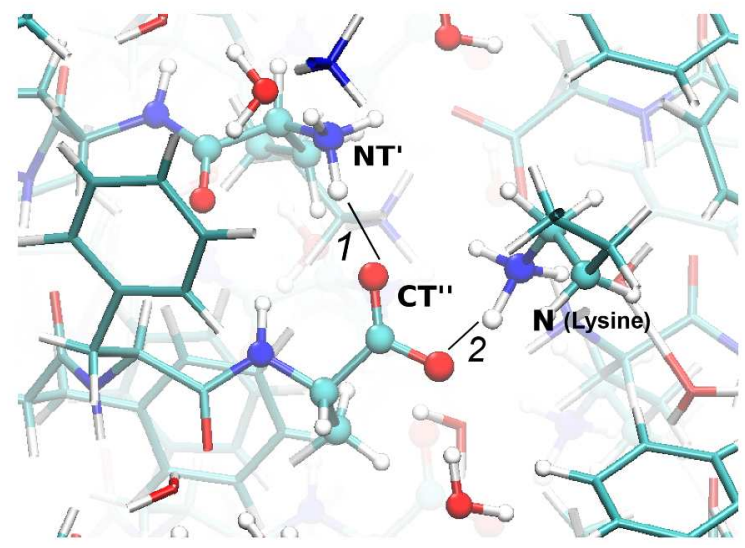

$238 \times 121 \mathrm{~mm}(300 \times 300$ DPI $)$ 
a)

i)

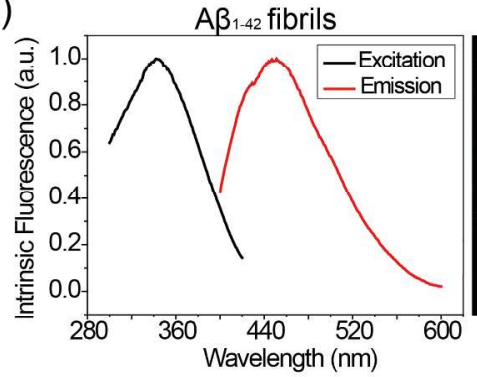

b)

i)

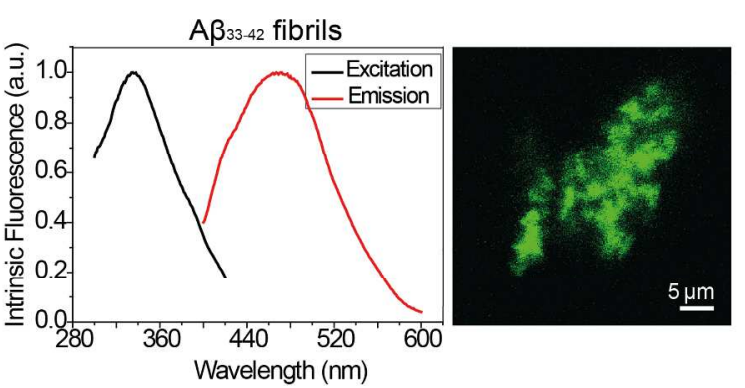

2 photon excitation

ii)

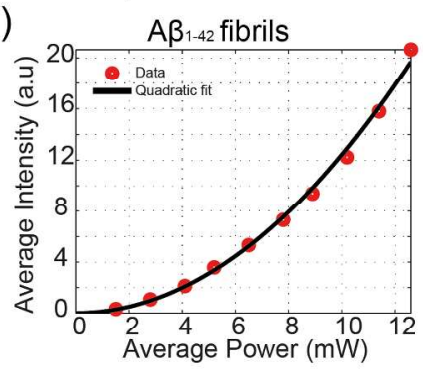

ii)

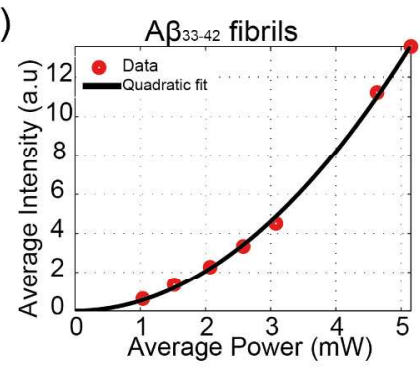

$214 \times 143 \mathrm{~mm}(300 \times 300 \mathrm{DPI})$ 
a)

i)

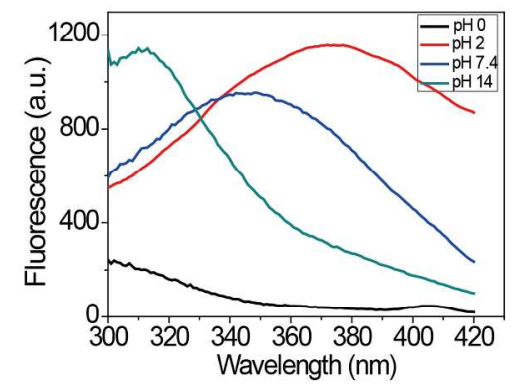

b)

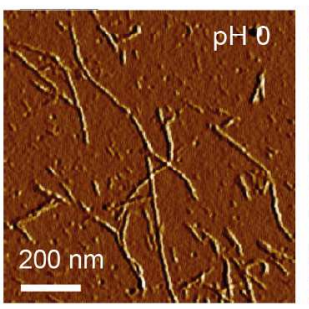

ii)
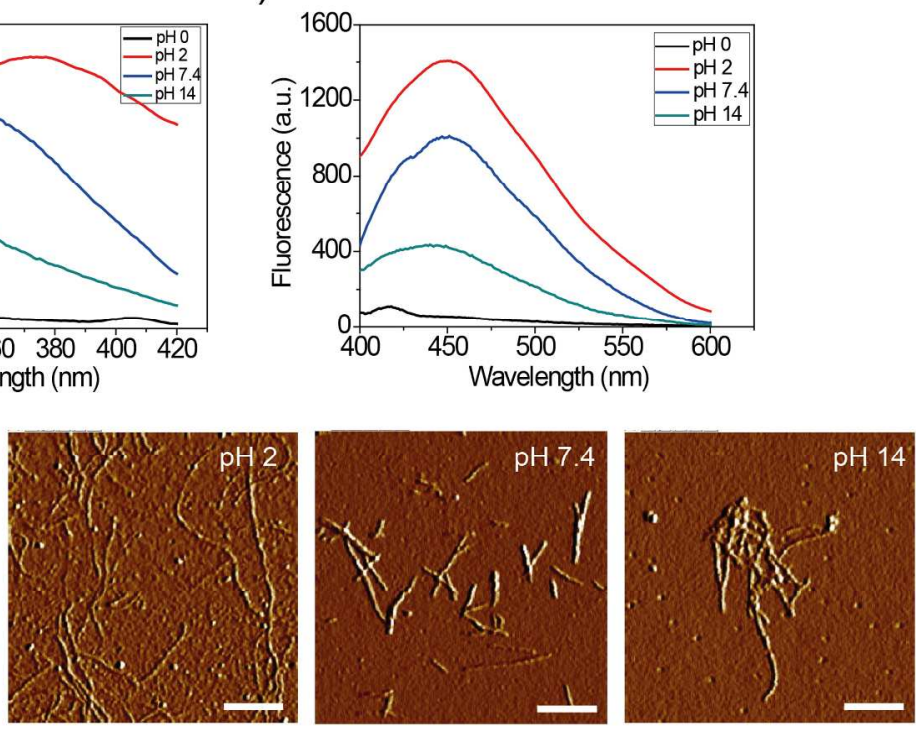

$198 \times 124 \mathrm{~mm}(300 \times 300 \mathrm{DPI})$ 
a)

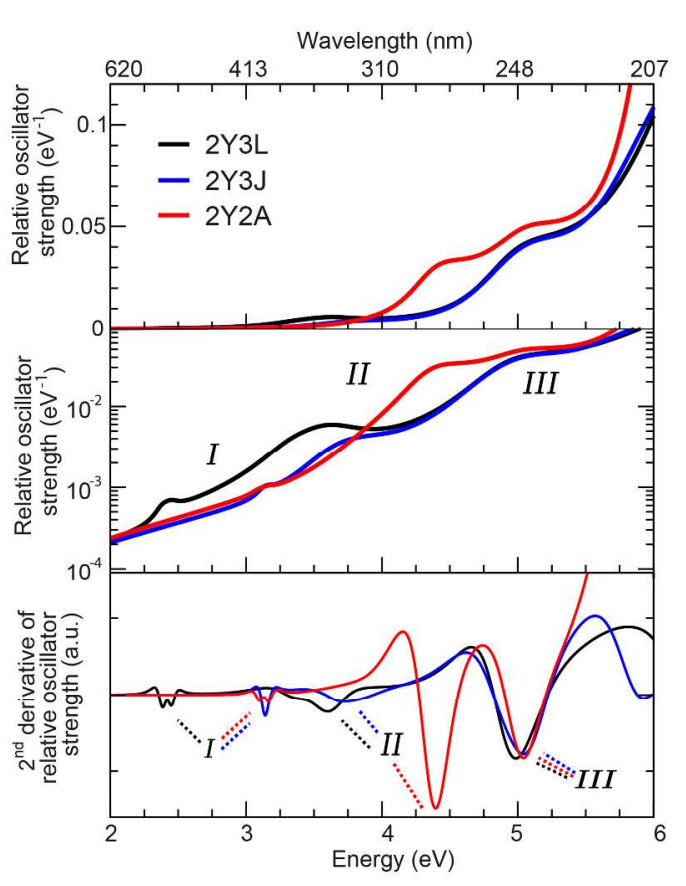

b)

2Y3J
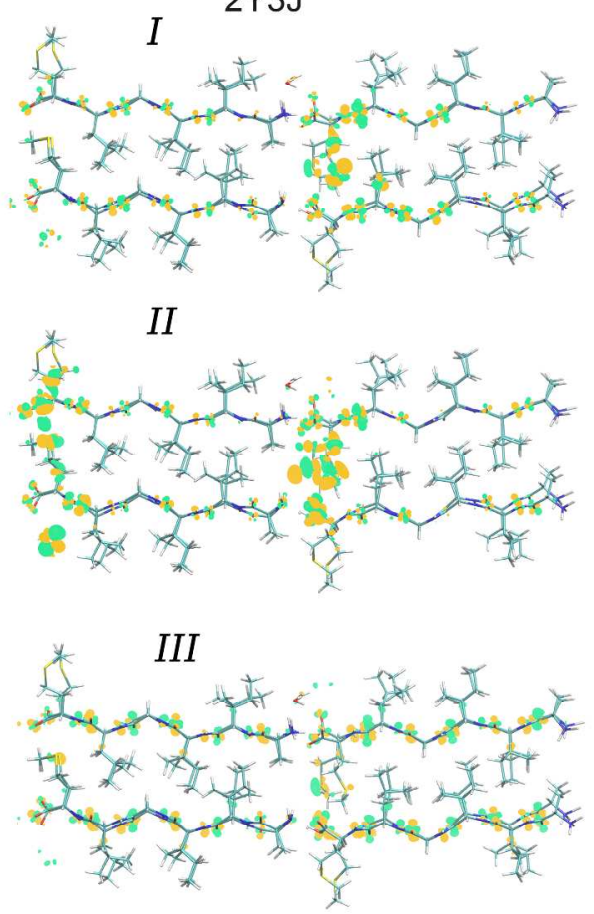

$238 \times 180 \mathrm{~mm}(300 \times 300$ DPI $)$ 
a)

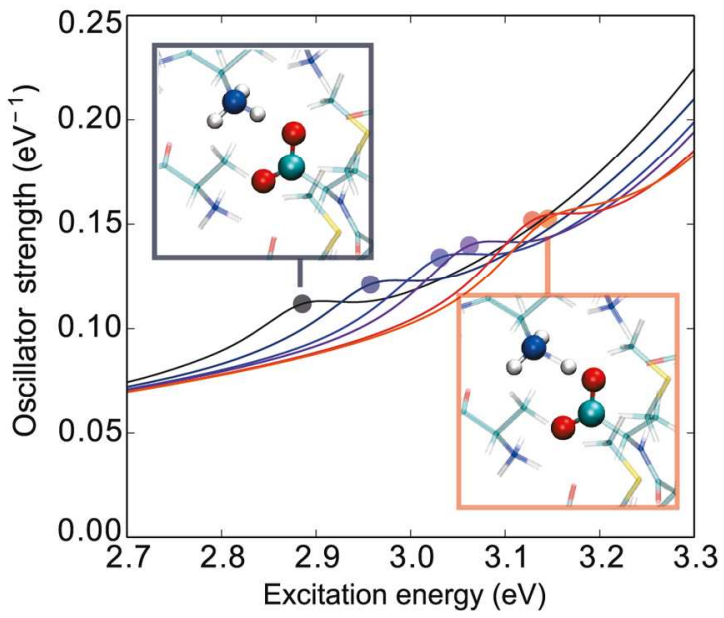

b)

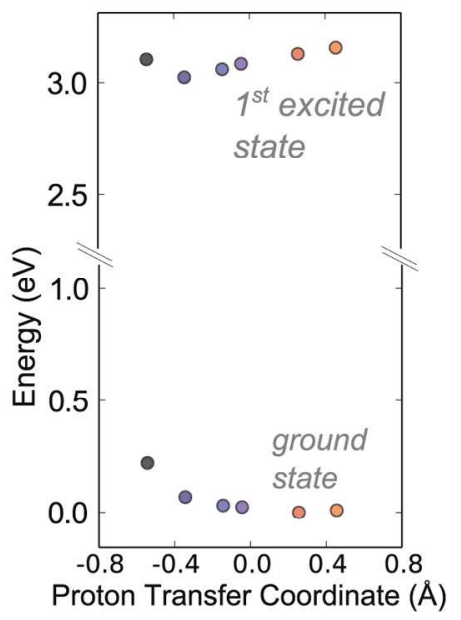

$153 \times 84 \mathrm{~mm}(300 \times 300$ DPI $)$ 


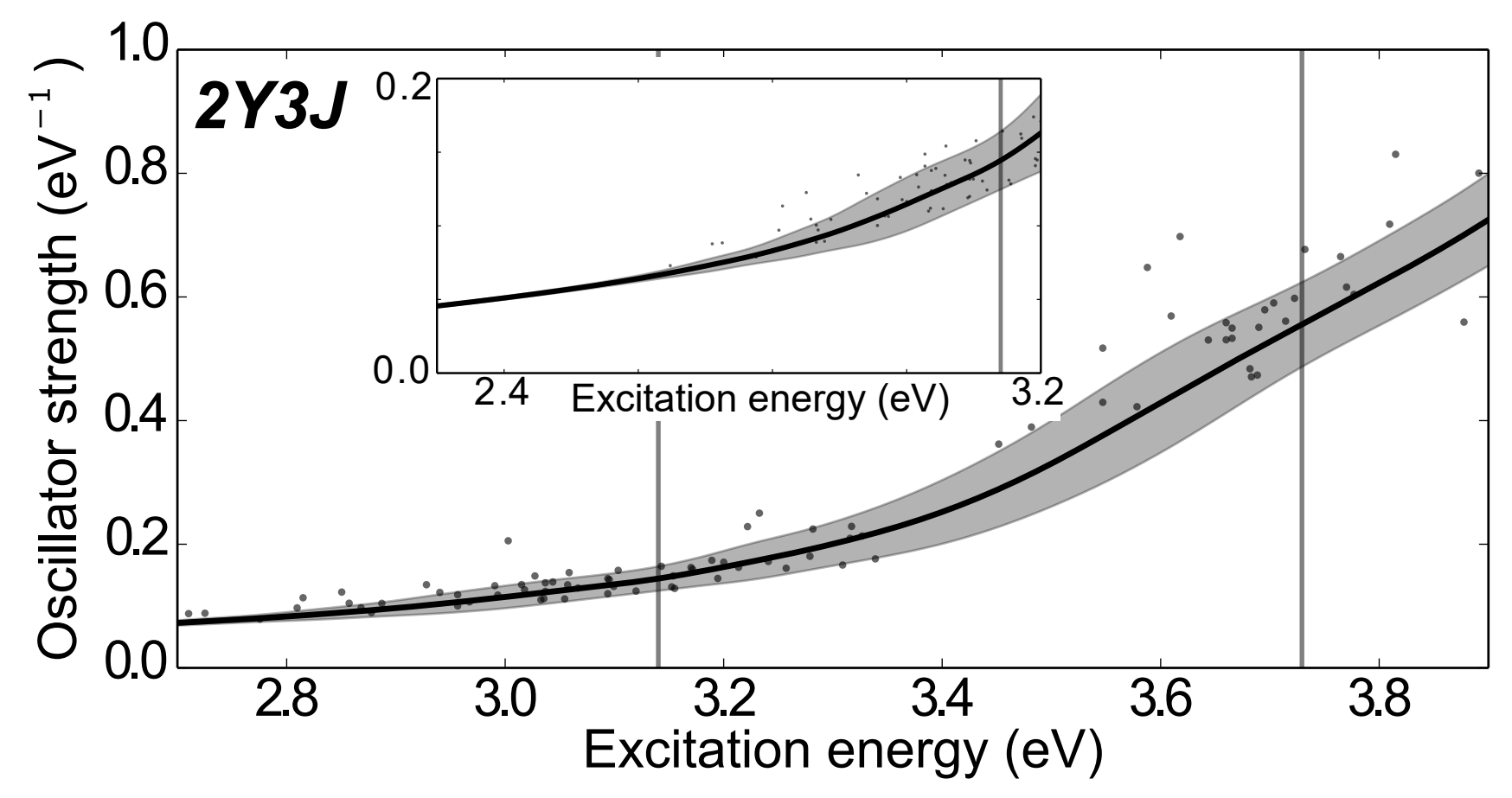

i)

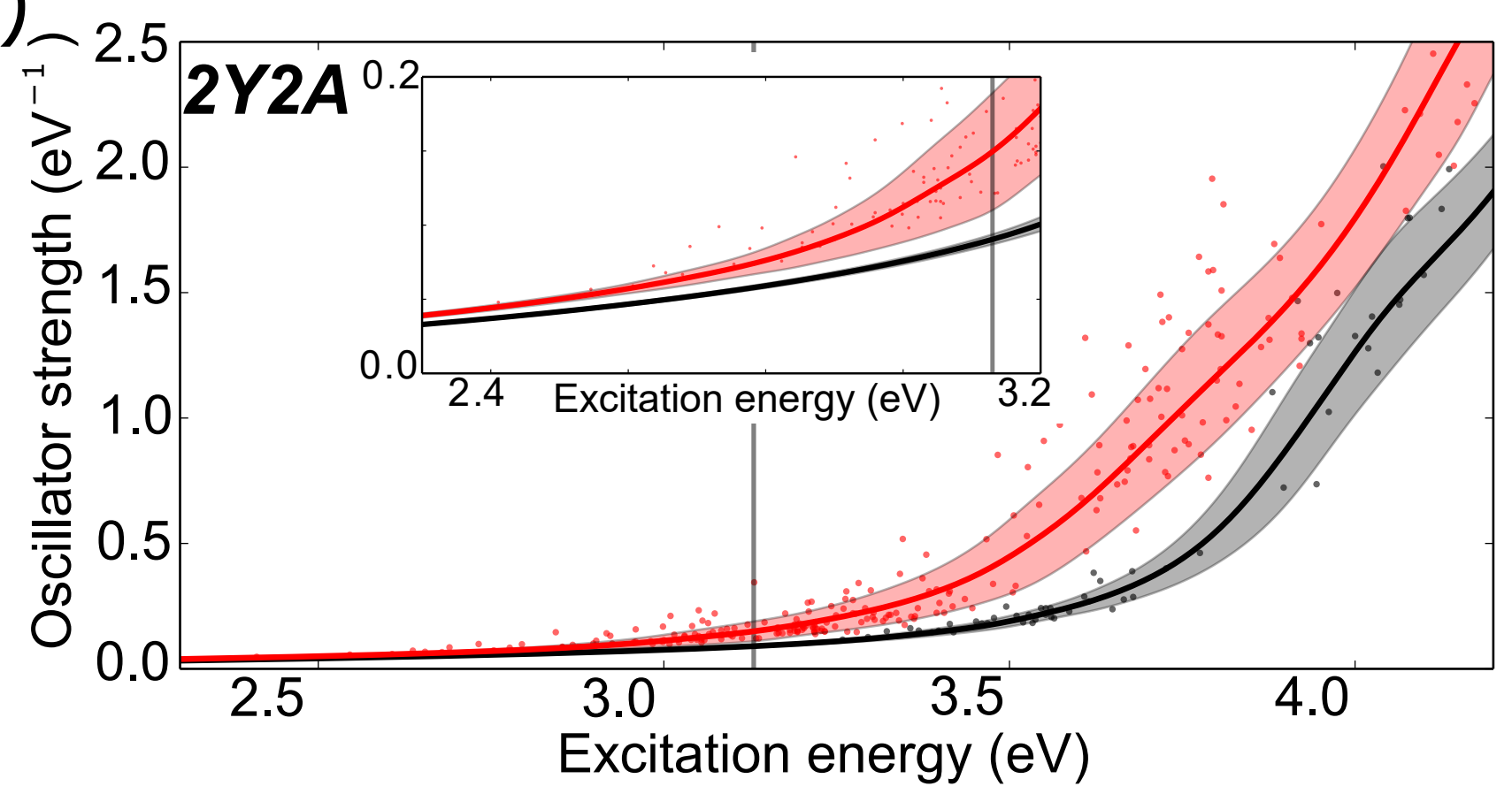

ii)

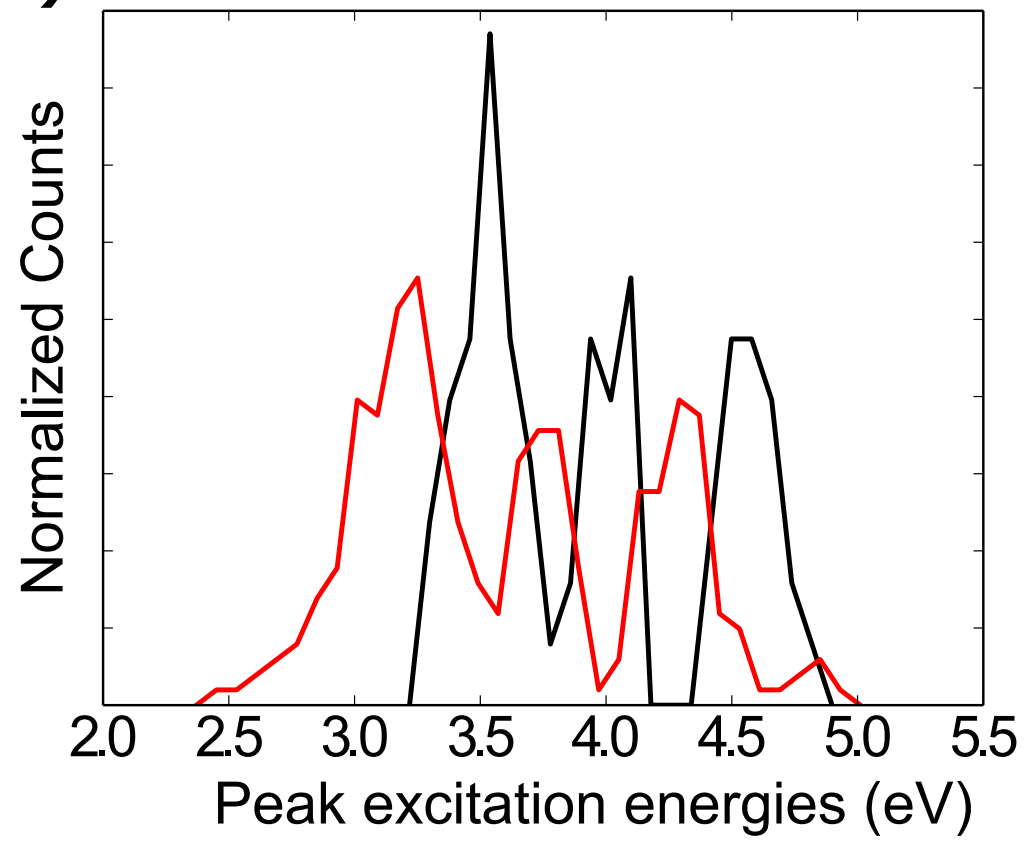


a)

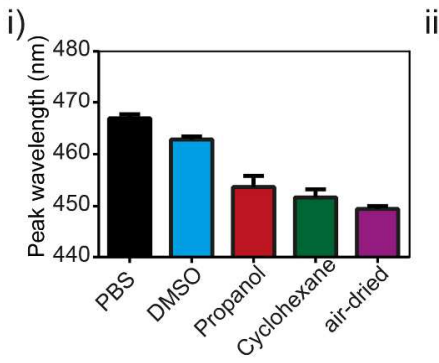

ii)

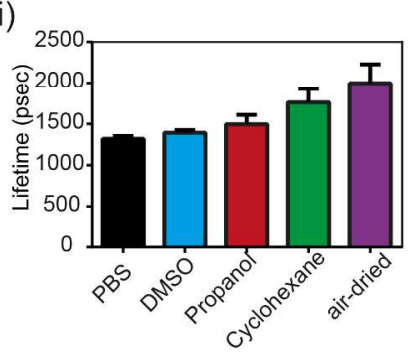

$245 \times 76 \mathrm{~mm}(300 \times 300 \mathrm{DPI})$ b)

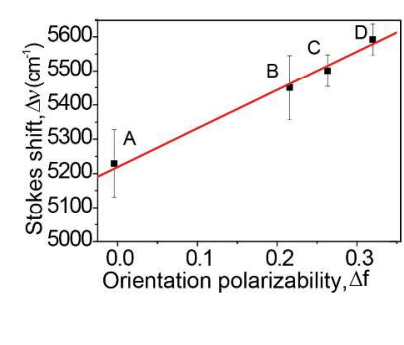


a)

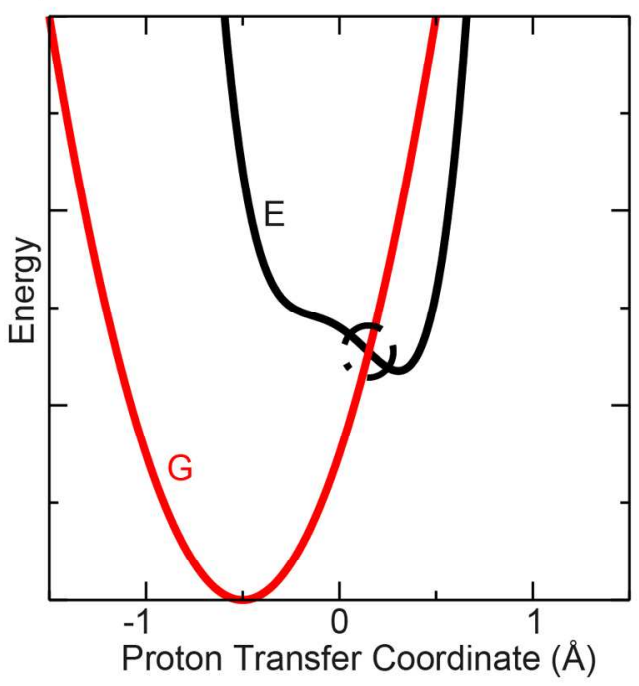

b)

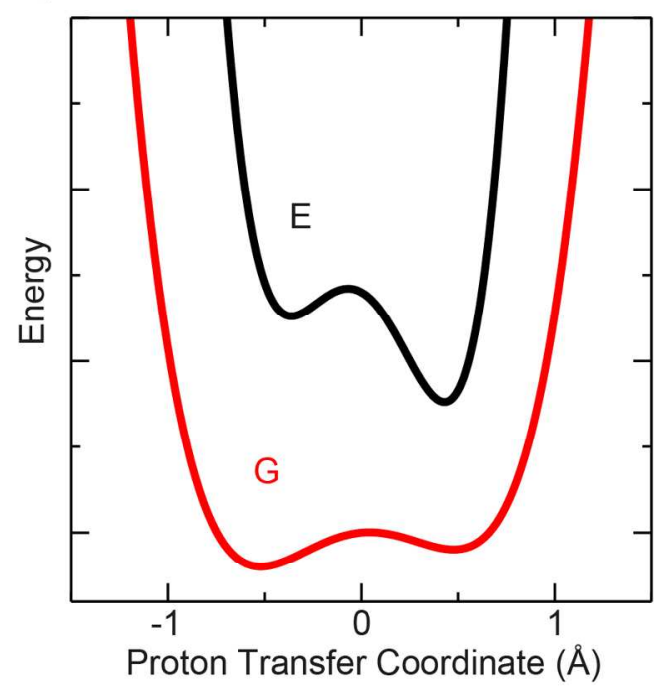

$159 \times 90 \mathrm{~mm}(300 \times 300 \mathrm{DPI})$ 


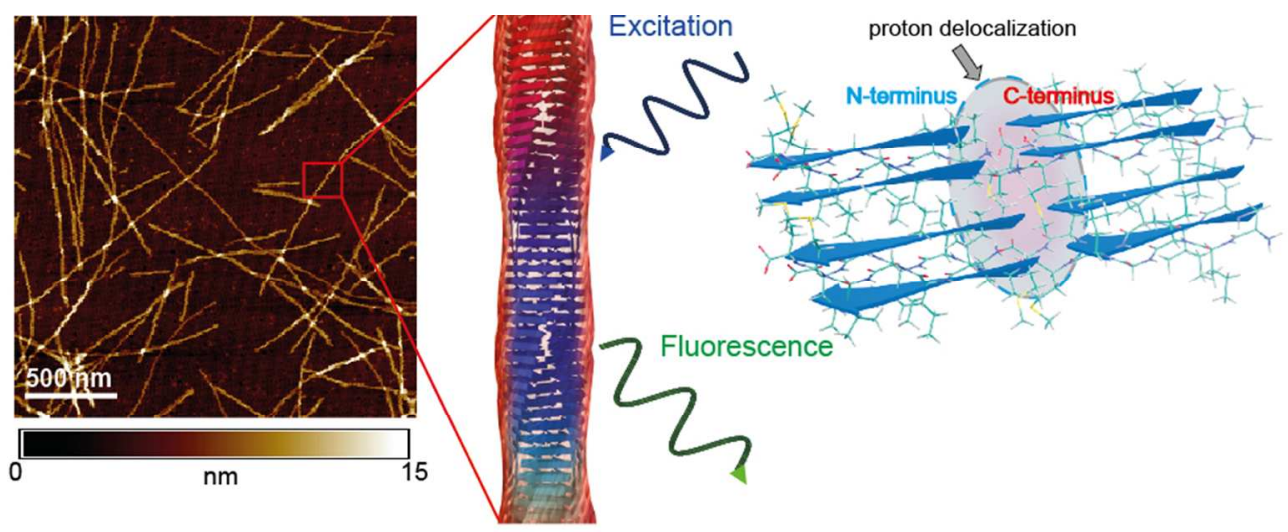

$83 \times 34 \mathrm{~mm}(300 \times 300$ DPI $)$ 